\title{
Dehydropeptide-based plasmonic magnetogels: a supramolecular composite nanosystem for multimodal cancer therapy
}

\begin{abstract}
Sérgio R. S. Veloso, ${ }^{a}$ J. A. Martins, ${ }^{b}$ Loic Hilliou, ${ }^{c}$ C. O. Amorim, ${ }^{d}$ V. S. Amaral, ${ }^{d}$ B. G. Almeida, ${ }^{\text {a Peter }}$ J. Jervis, ${ }^{b, e}$ Rute Moreira, ${ }^{e}$ David M. Pereira, ${ }^{e}$ Paulo J. G. Coutinho, ${ }^{a}$ Paula M. T. Ferreira, ${ }^{b}$ and Elisabete M. S. Castanheira, ${ }^{a} *$

Supramolecular hydrogels are highly promising for biomedical materials owing to the wide array of properties that can be tailored and modulated. The combination with plasmonic/magnetic nanoparticles into plasmonic magnetogels further improve its potential in biomedical applications, through the combination of complementary strategies, such as photothermia, magnetic hyperthermia, photodynamic therapy and magnetic-guided drug delivery. Here, a new dehydropeptide hydrogelator, Npx-L-Met-Z- $\triangle \mathrm{Phe}-\mathrm{OH}$, was developed and combined with two different plasmonic/magnetic nanoparticles architectures: core/shell manganese ferrite/gold nanoparticles or gold-decorated manganese ferrite nanoparticles with ca. $55 \mathrm{~nm}$ and $45 \mathrm{~nm}$ size, respectively. The characterization of magnetogels were performed by HR-TEM, FTIR, circular dichorism and rheological assays. The gels were tested as nanocarriers for a model antitumor drug, the natural compound curcumin. The incorporation of the drug in magnetogel matrices was confirmed through fluorescence-based techniques (FRET, fluorescence anisotropy and quenching). The curcumin release profiles were studied with and without excitation of the gold plasmon band. The transport of curcumin from magnetogels towards biomembrane models (small unilamellar vesicles) was assessed by FRET between the fluorescent drug and the lipid probe Nile Red. The developed magnetogels showed promising results for photothermia and photo-triggered drug release. The magnetogels bearing gold-decorated nanoparticles showed the best photothermia properties, while the ones containing core/shell nanoparticles had the best photoinduced curcumin release.
\end{abstract}

\section{Introduction}

The ongoing social, economic and cultural relation changes induced by the economic transition in low-income countries and the adoption of unhealthy lifestyles in high-income countries contribute to cancer keeping as a leading cause of death worldwide, with lung, colorectal, breast and prostate exhibiting the highest incidence ratio. $^{1-6}$ Conventional chemotherapy has only shown improved survival rates on early-stage disease in high-income countries, which can be accounted for the lack of specificity, therapeutic effectiveness and cost, besides worsening patient's quality of life. ${ }^{6,7}$ Supramolecular hydrogels are stimulus-responsive self-assembled intertwined fibrillar structures achieved through the cooperative effect of different non-covalent intermolecular interactions: hydrogen bonding, van der Waals, electrostatic, hydrophobic and

\footnotetext{
a. Centro de Física (CFUM), University of Minho, Campus de Gualtar, 4710-057 Braga, Portugal.

b. Centro de Química (CQUM), University of Minho, Campus de Gualtar, 4710-057 Braga, Portugal.

c. Institute for Polymers and Composites/I3N, Department of Polymer Engineering, University of Minho, Campus de Azurém, 4800-058 Guimarães, Portugal.

d. Physics Department and CICECO, University of Aveiro, Campus de Santiago, 3810-193 Aveiro, Portugal. Laboratory of, Department of Chemistry, Faculty of Pharmacy, University of Porto

e. REQUIMTE/LAQV, Lab. of Pharmacognosy, Dep. of Chemistry, Faculty of Pharmacy, University of Porto, R. Jorge Viterbo Ferreira, 228, 4050-313 Porto, Portugal.

+ Corresponding author; Phone: +351253604321; Fax: +351253604061; ecoutinho@fisica.uminho.pt

Electronic Supplementary Information (ESI) available: Detailed synthesis procedure; ${ }^{1} \mathrm{H}$ NMR spectra; UV-Vis absorption spectra; Critical aggregation assays; Docking in COX1; Details of XRD analysis; Mechanical spectra; FTIR spectra; EDX spectra; Nanoparticle size histograms from TEM; FFT of TEM image; Spectral overlap for FRET assays; Deduction of eqn. (7); Data of drug release assays. See DOI: 10.1039/x0xx00000x
}

aromatic interactions. ${ }^{8-18}$ The microdomains of solvent pockets afford physical-chemical similarity to the cell matrix, but also allow the retention of nanoparticles, which tailors and modulates the hydrogel matrix structure and mechanical, electrical, optical, and thermal properties. ${ }^{10-17,19}$ The hydrophobic and hydrophilic microdomains allow the loading and efficient transport of a wide variety of drugs, reducing side effects and enabling higher doses in therapy. ${ }^{20}$

The combination of hydrogels with magnetic nanoparticles affords magnetogels, providing control and targeting of the nanosystem to a specific location through a magnetic field gradient and a higher therapeutic efficiency, owing to the synergistic effect between magnetic hyperthermia and enhanced drug release promoted by the application of an alternating magnetic field (AMF). ${ }^{21-25}$ The higher magnetic susceptibility of manganese ferrite, when compared to other transition metal ferrites, makes it an appealing material for theranostic applications. ${ }^{26,27}$ Moreover, coating with gold might afford higher biocompatibility and lower toxicity, together with the surface plasmon resonance (SPR) phenomenon that allows the conversion of electromagnetic radiation into heat. ${ }^{28-32}$ Hereby, core/shell nanoparticles afford a high therapeutic potentiality owing to the combination of different strategies such as photothermia, photodynamic therapy, magnetic hyperthermia and magnetic-guided drug delivery.

In this work, gold-decorated manganese ferrite and core/shell manganese ferrite/gold nanoparticles were synthesized, characterized and incorporated into a new dehydrodipeptide hydrogel (Npx-L-Met-Z- $\triangle \mathrm{Phe}-\mathrm{OH}$, compound $\mathbf{1}$ ) containing naproxen 
(Npx) and methionine (Met), to avert the leashing out of nanoparticles through the S-Au bond. The dehydroamino acid dehydrophenylalanine ( $\Delta \mathrm{Phe}$ ) endows the peptide hydrogelator with proteolytic stability and adds conformational restraints to the peptide backbone, while the naproxen group affords selectivity towards cyclooxygenase. ${ }^{11,33,34}$ The mechanical properties of the magnetogels were assessed and the effect of the nanoparticles on the hydrogel fibrillar matrix was investigated. Moreover, the magnetogels were evaluated on the loading of a hydrophobic model drug (curcumin). The interaction with biomembrane models was studied as well as the use of photothermia as a trigger to accelerate curcumin release.

The proposed multifunctional nanosystem is promising for cancer therapy owing to the potential tunability of the mechanical properties, controlled drug delivery to biomembrane models through photothermia, besides the proteolytic stability, anti-inflammatory properties and magnetically-guided drug delivery.

\section{Experimental}

\section{Synthesis procedure of the hydrogelator}

General methods. Compound $\mathbf{1}$ was prepared by synthetic methodologies (see Supplementary Information for detailed description) developed in previous works ${ }^{35,36}$ and fully characterized by ${ }^{1} \mathrm{H}$ and ${ }^{13} \mathrm{C}$ NMR spectroscopy (NMR) and High Resolution Mass Spectrometry (HRMS). ${ }^{1} \mathrm{H}$ and ${ }^{13} \mathrm{C}$ NMR spectra were recorded on a Bruker Avance III at 400 and $100.6 \mathrm{MHz}$, respectively. DEPT $\theta 45^{\circ}$ and $135^{\circ}, \mathrm{HMQC}$ and $\mathrm{HMBC}$ were used to attribute some signals. Chemical shifts $(\delta)$ are reported in parts per million (ppm) and coupling constants $(\mathrm{J})$ are reported in Hertz $(\mathrm{Hz})$. Petroleum ether refers to the boiling range of $40-60{ }^{\circ} \mathrm{C}$. Acetonitrile was dried over silica and calcium hydride $\left(\mathrm{CaH}_{2}\right)$, while dichloromethane was dried over calcium hydride, and then distilled and stored over molecular sieves. Melting points $\left({ }^{\circ} \mathrm{C}\right)$ were determined in a Gallenkamp apparatus and are uncorrected.

\section{Synthesis procedure of magnetic/plasmonic nanoparticles}

Preparation of manganese ferrite nanoparticles. Manganese ferrite nanoparticles were synthesized by the co-precipitation method in a solution with a total volume of $5 \mathrm{~mL}$, as described by Rodrigues et al. ${ }^{37}$ Initially, a mixture of $500 \mu \mathrm{L}$ of $\mathrm{MnSO}_{4} \cdot \mathrm{H}_{2} \mathrm{O} 0.5 \mathrm{M}$ aqueous solution and $500 \mu \mathrm{L}$ of $\mathrm{FeCl}_{3} .6 \mathrm{H}_{2} \mathrm{O} 1 \mathrm{M}$ was prepared and added, drop by drop, to an aqueous solution with $612 \mu \mathrm{L}$ of $\mathrm{NaOH}$ $50 \%$ at $90{ }^{\circ} \mathrm{C}$, with constant magnetic stirring. After $2 \mathrm{~h}$ at $90{ }^{\circ} \mathrm{C}$, nanoparticles were formed, and purification was carried out by repeated centrifugations, dispersion in deionized water, and drying at $100{ }^{\circ} \mathrm{C}$.

Preparation of core-shell manganese ferrite/gold nanoparticles. The synthesis of gold-coated manganese ferrite nanoparticles was adapted from a previously reported procedure. ${ }^{38}$ Thus, a $5 \mathrm{~mL}$ aqueous solution of $\mathrm{MnFe}_{2} \mathrm{O}_{4}$ nanoparticles ( $40 \mathrm{mg}$ ) was added to $25 \mathrm{~mL}$ of glycerol and left stirring until $200{ }^{\circ} \mathrm{C}$, in a silicone oil bath. A solution of gold(III) chloride hydrate, $\mathrm{HAuCl}_{4} \cdot 3 \mathrm{H}_{2} \mathrm{O}(2 \mathrm{~mL}, 0.04 \mathrm{M})$ was added dropwise and stirring was kept for $15 \mathrm{~min}$. The mixture was washed with ethanol through centrifugation, followed by magnetic separation and left to dry at $100^{\circ} \mathrm{C}$.

Preparation of gold nanoparticles functionalized with 3-mercaptopropionic acid. The synthesis of gold nanoparticles functionalized with 3-mercaptopropionic acid (MPA) followed a procedure adapted from a previously reported method. ${ }^{39} 60 \mathrm{mg}$ of gold(III) chloride hydrate, $\mathrm{HAuCl}_{4} .3 \mathrm{H}_{2} \mathrm{O}$, were dissolved in ethanol $(50 \mathrm{~mL})$, followed by the addition of 3-mercaptopropionic acid (3.5 $\mathrm{mg})$ under vigorous stirring. A solution of $\mathrm{NaBH}_{4}(50 \mathrm{mg})$ in $\mathrm{NaOH} 1$ $\mathrm{M}(3 \mathrm{~mL})$ was added dropwise and the mixture was left stirring for $30 \mathrm{~min}$. A black/brown precipitate was formed, and purification was attained by centrifugation and washing with ethanol. The product was dried and stored in the freezer.

Preparation of gold-decorated manganese ferrite nanoparticles. The $\mathrm{MnFe}_{2} \mathrm{O}_{4}$ nanoparticles ( $9 \mathrm{mg}$ ) were washed with $\mathrm{NaOH} 1 \mathrm{M}$, dried and dispersed in DMSO $(5 \mathrm{~mL})$. Then, CDI (1,1'-carbonyldiimidazole) ( $63.36 \mathrm{mg}$ ) was added and the reaction was left for $2 \mathrm{~h}$ under vigorous stirring above $60^{\circ} \mathrm{C}$. The mixture was washed by repeated centrifugation and addition of DMSO. The CDI-activated magnetic nanoparticles were dispersed in DMSO and, then, MPA-functionalized gold nanoparticles $(35.7 \mathrm{mg}$ ) were added. The reaction was left for $1 \mathrm{~h}$ under vigorous stirring above $60^{\circ} \mathrm{C}$. The product of the reaction was obtained by magnetic separation. The nanoparticles were purified by repeated centrifugation and washed with DMSO. The gold-decorated nanoparticles were further functionalized with 2-aminoethanol $(0.2 \mathrm{~g})$ using the CDI $(63.33 \mathrm{mg})$ coupling procedure described above. The product was magnetically separated and washed with water to remove the reagents and DMSO.

\section{Self-assembly of hydrogel}

pH dependence. The weighted mass of hydrogelator was dissolved in the required $\mathrm{pH}$ buffer, previously prepared from a sodium phosphate $0.1 \mathrm{M}$ solution and a mixed solution of citric acid $(0.05 \mathrm{M})$ and boric acid $(0.2 \mathrm{M})$, as described by Perrin and Dempsey. ${ }^{40}$ Hydrogelator solutions at $0.2 \mu \mathrm{M}$ concentration were obtained in the $\mathrm{pH}$ range $2-10$. The fluorescence emission spectra were measured for each solution to evaluate the $\mathrm{pH}$ influence on the hydrogelator behaviour.

Critical aggregation concentration. The critical aggregation concentration was assessed through titration of the hydrogelator in the respective $\mathrm{pH}$ buffer. The concentration range to consider was determined by sequentially preparing $10 \times$ diluted solutions and the range was chosen according to where a fluorescence increase, or decrease, was observed.

Critical gelation concentration. To determine the critical gelation concentration through fluorescence spectroscopy, aqueous solutions of the hydrogelator 1 in a concentration range 0.2-0.8 $\mathrm{wt} \%$ were prepared. The $\mathrm{pH}$ was chosen according to the results obtained in the self-assembly study at different $\mathrm{pH}$ values. Self-assembly was attained by dissolving the hydrogelator in basic $\mathrm{pH}$ by adding $\mathrm{NaOH} 1 \mathrm{M}$ and then glucono- $\delta$-lactone $(\mathrm{GdL})$ was added to decrease $\mathrm{pH}$ homogeneously, sonicating and left stabilizing. 
Development of magnetogels. The prepared nanoparticles were added to the hydrogel at a final volume of $200 \mu \mathrm{L}$ and at the required concentration ( $\mathrm{m} / \mathrm{m} \%$ relatively to the hydrogelator mass) from a starting solution at 2 wt\%. All hydrogel/magnetogel solutions were left standing at room temperature until gel phase was attained.

Incorporation of curcumin and transport to biomembrane models. The hydrogel and magnetogels were prepared, and curcumin was added for a final concentration of $2 \mu \mathrm{M}$. From the hydrogelator solution, $200 \mu \mathrm{L}$ were transferred to a fluorescence microcuvette and left standing until the hydrogel/magnetogel was formed. To study the interaction of hydrogels and magnetogels with biomembrane models, SUVs (small unilamellar vesicles) of egg phosphatidylcholine (egg-PC) and cholesterol 7:3 were prepared using the ethanolic injection technique. The lipid probe Nile Red ${ }^{41-43}$ was incorporated into the SUVs for a final concentration of $2 \mu \mathrm{M}$. All studies of drug incorporation and transport into SUVs were carried out by fluorescence-based techniques (fluorescence emission, FRET and fluorescence anisotropy).

\section{Spectroscopic measurements}

General methods. Fluorescence measurements were carried out using a Fluorolog 3 spectrofluorimeter, equipped with double monochromators in both excitation and emission, Glan-Thompson polarizers and a temperature-controlled cuvette holder. Fluorescence emission spectra were corrected for the instrumental response of the system. The excitation of the hydrogelators was set at $290 \mathrm{~nm}$ (naproxen), and the emission spectrum was collected between $300 \mathrm{~nm}$ and $550 \mathrm{~nm}$. Absorption spectra were recorded in a Shimadzu UV-3600 Plus UV-Vis-NIR spectrophotometer.

The fluorescence quantum yield, $\Phi_{s}$, can be determined by eqn (1) (standard method), ${ }^{44,45}$

$$
\Phi_{\mathrm{S}}=\frac{\left(A_{\mathrm{r}} F_{\mathrm{s}} n_{\mathrm{s}}^{2}\right)}{\left(A_{\mathrm{s}} F_{\mathrm{r}} n_{\mathrm{r}}^{2}\right)} \Phi_{\mathrm{r}}
$$

where $A$ is the absorbance at the excitation wavelength, $F$ is the integrated emission area and $n$ is the refraction index of the solvents. Subscripts $r$ and $s$ refer to reference and sample compound, respectively. The absorbance value at excitation wavelength was always less than 0.1 , in order to avoid inner filter effects. L-Tryptophan in aqueous buffer solution $(\mathrm{pH}=7.2)$ was used as reference $\left(\Phi_{\mathrm{r}}=0.14\right.$ at $\left.25^{\circ} \mathrm{C}\right) .{ }^{46}$

Fluorescence anisotropy measurements. The steady-state fluorescence anisotropy values, $r$, can be determined by eqn (2), ${ }^{47}$

$$
r=\frac{I_{\mathrm{VV}}-G I_{\mathrm{VH}}}{I_{\mathrm{VV}}+2 G I_{\mathrm{VH}}}
$$

where $I_{\mathrm{VV}}$ and $I_{\mathrm{VH}}$ are the intensities of the emission spectra obtained with vertical and horizontal polarization, respectively (for vertically polarized excitation light), $I_{\mathrm{HV}}$ and $I_{\mathrm{HH}}$ are the emission intensities obtained with vertical and horizontal polarization (for horizontally polarized excitation light) and $G=I_{\mathrm{HV}} / I_{\mathrm{HH}}$ is the instrumental correction factor.

FRET measurements. The drug incorporation into magnetogels network and interaction with biomembrane models was investigated by Förster Resonance Energy Transfer (FRET). FRET efficiency, $\Phi_{\text {FRET }}$, defined as the proportion of donor molecules that have transferred their excess energy to acceptor molecules, can be expressed by eqn (3), ${ }^{47}$

$$
\Phi_{\mathrm{FRET}}=1-\frac{I_{D A}}{I_{D}}
$$

where $I_{D A}$ and $I_{D}$ are the donor integrated fluorescence intensities in the presence and absence of acceptor, respectively. FRET efficiency can also be related with donor-acceptor intermolecular distance, $R_{D A}$, and the Förster radius (critical diameter), $R_{0}$, through the eqn (4), ${ }^{47}$

$$
\Phi_{\mathrm{FRET}}=\frac{1}{1+\left(\frac{R_{D A}}{R_{0}}\right)^{6}}
$$

Förster radius, $R_{0}$, the distance at which FRET efficiency is $50 \%$, can be determined by the spectral overlap, $J(\lambda)$, between the donor fluorescence emission and the acceptor absorption, according to eqn (5) and eqn (6) (with $R_{0}$ in $\AA, \lambda$ in nm, $\varepsilon_{A}(\lambda)$ in $\mathrm{M}^{-1} \mathrm{~cm}^{-1}$ ), ${ }^{47}$

$$
\begin{gathered}
R_{0}=0.2108\left[\kappa^{2} \Phi_{D} n^{-4} J(\lambda)\right]^{1 / 6} \\
J(\lambda)=\int_{0}^{\infty} I_{D}(\lambda) \varepsilon_{A}(\lambda) \lambda^{4} d \lambda
\end{gathered}
$$

where $\kappa^{2}=2 / 3$ is the orientational factor assuming random orientation of the dyes, $\Phi_{D}$ is the donor fluorescence quantum yield in the absence of energy transfer, $n$ is the refraction index of the medium, $I_{D}(\lambda)$ is the fluorescence spectrum of the donor normalized so that $\int_{0}^{\infty} I_{D}(\lambda) d \lambda=1$, and $\varepsilon_{A}(\lambda)$ is the molar absorption coefficient of the acceptor.

Transmission Electron Microscopy (TEM). HR-TEM images were recorded using a Transmission Electron Microscope JEOL JEM 2010F operating at $200 \mathrm{kV}$ coupled to an Electron Dispersive Spectroscopic analyzer (EDS) at C.A.C.T.I (Centro de Apoio Cientifico e Tecnolóxico á Investigación), Vigo, Spain. The samples were prepared according to the uranyl acetate staining method. After preparation of the hydrogel and magnetogel solutions, through prior dissolution at $\mathrm{pH}=10$ followed by addition of $\mathrm{GdL}$, a drop of each sample was placed onto a TEM 400 mesh copper grid with Formvar/Carbon (ref. S162-4 from Agar Scientific), held by tweezers and the excess solution was cleaned. A droplet of uranyl acetate was placed over the sample, the excess solution was cleaned, and the grid was left to dry. The processing of TEM images was performed using ImageJ software, which consisted in enhancing local contrast and adjusting brightness followed by selection of particles and fibres.

X-Ray Diffraction. A conventional PAN'alytical X'Pert PRO diffractometer was used for X-ray diffraction (XRD) analyses, operating with $\mathrm{Cu} \mathrm{K}_{\alpha}$ radiation, in a Bragg-Brentano configuration.

FTIR measurements. Fourier Transform Infrared Spectroscopy (FTIR) measurements with Attenuated Total Reflection (ATR) were performed in a Bruker $66 \mathrm{~V}$ Spectrometer, with a resolution of $1 \mathrm{~cm}^{-1}$. The amide I region sensitivity to secondary structure was explored to assign the secondary structures. However, the variety of frequencies leads to a featureless band consisting of various component bands that are instrumentally unresolved. Thus, the second-derivative was used to identify overlapping bands and assign the corresponding bands, as it does not require any kind of arbitrary half-bandwidths and enhancement factors. ${ }^{48-52}$ Hereby, a first approach was the direct curve fitting to the amide I after 
baseline correction; the second method was curve fitting to the second derivative peaks; and the third one was the curve fitting to amide III region (1220-1330 $\mathrm{cm}^{-1}$ ), that is mainly contributed by the in-phase combination of $\mathrm{N}-\mathrm{H}$ in-plane bending and $\mathrm{C}-\mathrm{N}$ stretching vibration. The amide I band is affected by the water absorption overlapping, which not only mislead the secondary structure percentages, but also worsens the ambiguity in the assignment of random coil $\left(1642-1657 \mathrm{~cm}^{-1}\right)$ and $\alpha$-helix $\left(1648-1657 \mathrm{~cm}^{-1}\right)$ bands. ${ }^{52}$ Hereby, the amide I region quantitative analysis was carried out considering that $\alpha$-helix does not have a significant contribution when dealing with small structures, and that only $\beta$-sheet, random coil and turns are present. The amide III region does not suffer from the water absorption signal and has a better localization of the different contributions of amide bond vibrations. However, amide III lacks its accuracy due to the overlapping of other vibrational groups and the weak distinction between random coil $\left(1270-1255 \mathrm{~cm}^{-1}\right)$ and turn $\left(1295-1270 \mathrm{~cm}^{-1}\right)$ frequencies.

Circular dichroism. The $\mathrm{CD}$ spectra were recorded at $20{ }^{\circ} \mathrm{C}$ on a Jasco model J-1500 spectropolarimeter, under a constant flow of nitrogen gas. Peptide hydrogelator $0.02 \mathrm{wt} \%$ solutions were loaded into $0.1 \mathrm{~mm}$ quartz cells. Spectra were acquired with $1 \mathrm{~nm}$ steps, $1 \mathrm{~nm}$ bandwidth and 1 second collection time per step, taking three averages. The obtained data were smoothed by an 11-point Savitsky-Golay filter to remove random noise elements from the averaged spectra.

Molecular docking studies of the hydrogelator. The crystal structure of Mus musculus cyclooxygenase 2 (COX-2) expressed in Spodoptera frugiperda complexed with naproxen (PDB code: 3NT1) and Ovies aries cyclooxygenase 1 (COX-1) expressed in Spodoptera frugiperda in complex with flurbiprofen (PDB code: $3 \mathrm{~N} 8 \mathrm{Z}$ ) were used as the protein receptor models. The optimized geometries of the hydrogelator ground state were obtained from $a b$ initio molecular quantum chemistry calculations with Gaussian 09 software, $^{53}$ and with the use of ONIOM method, setting the naproxen moiety for the high level with a $6-311+G(d, p)$ basis set at the DFT B3LYP level of theory and the peptide backbone for the low level at 3-21G basis set and HF B3LYP level of theory in gas phase. Docking of the receptor protein with the hydrogelator was performed using AutoDockTools-1.5.6 Software Genetic Algorithm. The calculation was set up to 50 runs, 270000 maximum number of generations, 2500000 maximum number of energy evaluations, and $60 \times 60 \times 60$ grid points for $3 \mathrm{NT} 1$ and $3 \mathrm{~N} 8 \mathrm{Z}$ with $0.375 \AA$ spacing. The lowest docked energy conformation with naproxen moiety in the active site was chosen as the best conformation. Visualization of the complex protein-ligand interactions was analysed with PyMOL software.

Magnetic properties. Magnetization measurements were done in a MPMS3 SQUID magnetometer (Quantum Design). The hysteresis cycles (magnetization vs. magnetic field) of the samples were measured in the convenient field range for each sample. A specific magnetic field correction for the trapped flux in the superconducting coil was made achieving an accuracy of residual less than 2 Oe.

Rheology. The viscoelastic characterization of gels was performed with a stress-controlled rotational rheometer (MCR300, Anton
Paar). Liquid samples were loaded into the Couette geometry of the rheometer and temperature was kept at $25{ }^{\circ} \mathrm{C}$ during testing. After a three hours rest period, ensuring gel setting and structural equilibrium of samples, gels mechanical spectra were recorded using a fixed strain amplitude (values ranging from $10^{-4}$ to $10^{-2} \%$ depending on the gel elasticity) and ramping the frequency from $100 \mathrm{~Hz}$ down to $0.01 \mathrm{~Hz}$. Finally, a sweep in the strain amplitude was performed from $0.001 \%$ to $500 \%$, to assess the linear regime of viscoelasticity and the large amplitude oscillatory strain (LAOS) regime. Experiments were repeated with softer gels in order to improve the data from the frequency sweep, using larger strain amplitudes while still belonging to the linear regimes assessed by previous strain sweeps.

Photothermia in magnetogels. Hydrogels containing curcumin $(2 \mu \mathrm{M})$ were subjected to increasing temperatures to measure the dependence of model drug fluorescence emission on temperature. Hydrogels/magnetogels loaded with curcumin were irradiated with a setup consisting on a Xenon arc lamp (200 W) and an optical fibre, using a Thorlabs FEL0600 long pass filter with cut-on wavelength at $600 \mathrm{~nm},{ }^{38}$ to ensure no curcumin excitation. The detection was attained using a SPEX Fluorolog 2 spectrofluorimeter for 5 hours. At the fifth hour, the irradiation was stopped and fluorescence emission was measured one hour afterwards. The dependence of fluorescence emission on temperature was used to estimate the local temperature created by excitation on plasmon band of the irradiated magnetogels.

Drug release to $\mathrm{pH}=7$ buffer and biomembrane models. Hydrogel and magnetogel loaded with $20 \mu \mathrm{M}$ curcumin were prepared. A solution of egg-PC/cholesterol SUVs (model membranes) was added to the drug-loaded hydrogel or magnetogel. The curcumin accumulation into SUVs was monitored for 8 hours, with and without irradiation of the magnetogel.

The release of curcumin to $\mathrm{pH}=7$ buffer followed a similar procedure as described for biomembrane models (SUVS). Hydrogels/magnetogels loaded with $20 \mu \mathrm{M}$ curcumin were prepared and left stabilizing overnight. The hydrogel/magnetogel was washed five times with $1 \mathrm{~mL}$ of $\mathrm{pH}=7$ buffer and water $(1.5 \mathrm{~mL})$ was added to the drug-loaded hydrogel or magnetogel. Aliquots of $300 \mu \mathrm{L}$ were taken, replaced with $\mathrm{pH}=7$ buffer and curcumin fluorescence emission was measured to determine the concentration at each time point.

Cytotoxicity assay. Cell viability was evaluated by the MTT reduction assay. ${ }^{54}$ Cells were cultured in 96 -well plates $(25,000$ cells/well) and allowed to attach for $24 \mathrm{~h}$. After incubation with Npx-L-Met-Z- $\triangle$ Phe-OH for $24 \mathrm{~h}$, MTT $(0.5 \mathrm{mg} / \mathrm{mL}$ final concentration) was added to each well and the plate was incubated for $75 \mathrm{~min}$ at $37^{\circ} \mathrm{C}$. Formazan crystals were dissolved by the addition of a DMSO:isopropanol mixture (3:1) and then quantified spectrophotometrically at $570 \mathrm{~nm}$ using a microplate reader (Multiskan Thermo Fisher Scientific). 


\section{Results and discussion}

Synthesis of the hydrogelator compound 1 . The hydrogelator methionyldehydrophenylalanine $\mathrm{N}$-protected with 2-(6-methoxy-2naphthyl)propionic acid (naproxen, Npx) was prepared using a conventional protocol in solution (Scheme 1).

The strategy for synthesis of hydrogelator Npx-L-Met-Z- $\triangle$ Phe-OH 1 involves the coupling of $\mathrm{N}$-tert-butyloxycarbonyl methionine (BocMet-OH, 2) with the methyl ester of $\beta$-hydroxyphenylalanine (H-D,LPhe $(\beta-\mathrm{OH})-\mathrm{OMe}, \quad 3)$, using the $N, N^{\prime}$-dicyclohexylcarbodiimide (DCC)/1-hydroxybenzotriazole (HOBt) procedure. Compound 4 was obtained as diastereomeric mixture in $84 \%$ yield. The reaction of the carboxylic acid of compound $\mathbf{3}$ gives an $\mathbf{O}$-acylurea. The latter is very reactive and can lead to the formation of several by-products such as $\mathrm{N}$-acylureas or oxazolones. ${ }^{55}$ Oxazolones are usually associated with epimerisation. The addition of 1-hydroxybenzotriazole to the reaction mixture reduces epimerization, through the formation of an active ester. ${ }^{55}$ Dehydration of the $\beta$-hydroxydipeptide 4 was attained by reaction with di-tert-butyl dicarbonate $\left(\mathrm{Boc}_{2} \mathrm{O}\right)$ in the presence of 4-dimethylaminopyridine (DMAP), followed by treatment with $N, N, N^{\prime}, N^{\prime}$ tetramethylguadinine (TMG). ${ }^{56}$

The dehydrodipeptide $\mathbf{5}$ was isolated in a $60 \%$ yield. The $\mathrm{N}$-tertbutoxycarbonyl group of $\mathbf{5}$ was removed with trifluoroacetic acid (TFA) and the $N$-deprotected dehydrodipeptide 6 was coupled with 2-(6-methoxy-2-naphthyl)propionyl chloride. The hydrogelator 1 was obtained after basic cleavage of the methyl ester of compound 7 (Figure S1 in Supplementary Information).

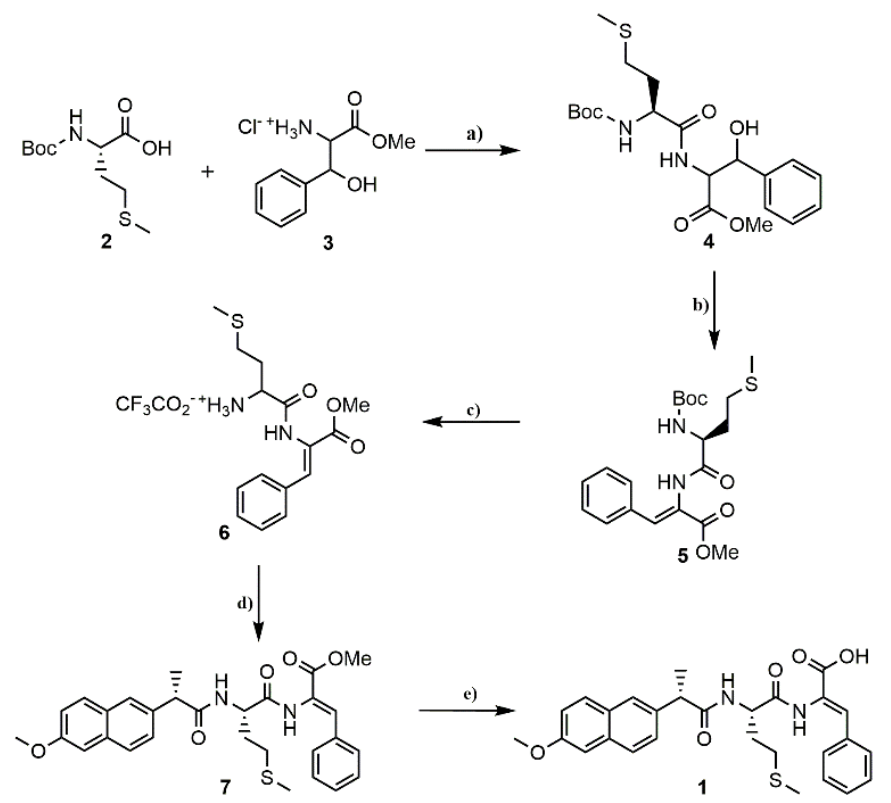

Scheme 1. Synthesis of hydrogelator 1. a) DCC, $\mathrm{HOBt}, \mathrm{Et}_{3} \mathrm{~N}, \mathrm{ACN}, \mathrm{rt}$; b) (1) $\mathrm{Boc}_{2} \mathrm{O}$, DMAP, dry ACN, rt (2) TMG; c) TFA; d) (S)-(+)-naproxen chloride, $\mathrm{Et}_{3} \mathrm{~N}, \mathrm{DCM}, \mathrm{rt}$; e) (1) $\mathrm{NaOH}(1 \mathrm{M}), 1$,4-dioxane, rt, (2) $\mathrm{KHSO}_{4}$.

\section{Characterization of the hydrogelator Npx-L-Met-Z- $\triangle \mathrm{Phe}-\mathrm{OH}$}

UV-visible absorption. The UV-visible absorption spectrum of the hydrogelator Npx-L-Met-Z- $\triangle \mathrm{Phe}-\mathrm{OH}$ shows the characteristic bands of naphthalene and naphthalene-based compounds ${ }^{36}$ (Figure S2 in Supplementary Information). The intense band between 210 and $240 \mathrm{~nm}$, with a peak at $233 \mathrm{~nm}$, is associated with the naphthalene long axis $\pi-\pi^{*}$ transition, while the medium band at $260-290 \mathrm{~nm}$ (with the absorption maximum at $273 \mathrm{~nm}$ ) is assigned to the naphthalene short axis $\pi-\pi^{*}$ transition. ${ }^{36,57}$ Moreover, the phenylalanine absorption, which has a maximum at $260 \mathrm{~nm},{ }^{58}$ and the peptide backbone $n-\pi^{*}$ and $\pi-\pi^{*}$ transition around $210-230 \mathrm{~nm}$ and $180-200 \mathrm{~nm}$, respectively, also contribute to the overall spectrum. ${ }^{36}$ The absorption spectrum is dominated by the naproxen moiety over phenylalanine, as expected considering the low molar absorption coefficient of the latter owing to a $n-\pi^{*}$ transition. ${ }^{58}$

Self-assembly parameters. Fluorescence spectroscopy was used to determine the $\mathrm{pH}$ dependence of hydrogelator self-assembly behaviour, critical gelation and aggregation concentration, by exploring the emission of intrinsic fluorophore naproxen. The hydrogelator solutions were excited at $290 \mathrm{~nm}$, as there is no direct excitation of dehydrophenylalanine, besides not changing the emission spectra, as the long-axis polarized state is the emitting state. ${ }^{58}$ The fluorescence emission spectrum of the hydrogelator shows a main band at $\lambda_{\max }=360 \mathrm{~nm}$ and a second fluorescence emission band with maximum around $450 \mathrm{~nm}$, which are associated to the naproxen monomer and aggregate, respectively (Figure 1A). ${ }^{36}$ The monomer fluorescence emission band shows little $\mathrm{pH}$ dependence in the range of $\mathrm{pH}$ values from 4 to 7 , which strongly increases at higher $\mathrm{pH}$, associated with the increasing deprotonated species, thus favouring the free monomer state. However, it should be noticed that, at $\mathrm{pH}=4$ and 5 , a large aggregate to monomer emission intensity ratio $\left(I_{2} / I_{1}\right)$ is attained, suggesting that self-assembly into aggregates is favourable at this $\mathrm{pH}$ value and decreases with increasing $\mathrm{pH}$. Moreover, using the MarvinSketch software, a predicted $\mathrm{p} K_{a}$ value of 3.7 was obtained for the terminal carboxylic acid, which is close to the $\mathrm{pH}$ where $I_{2} / I_{1}$ maximum is verified.

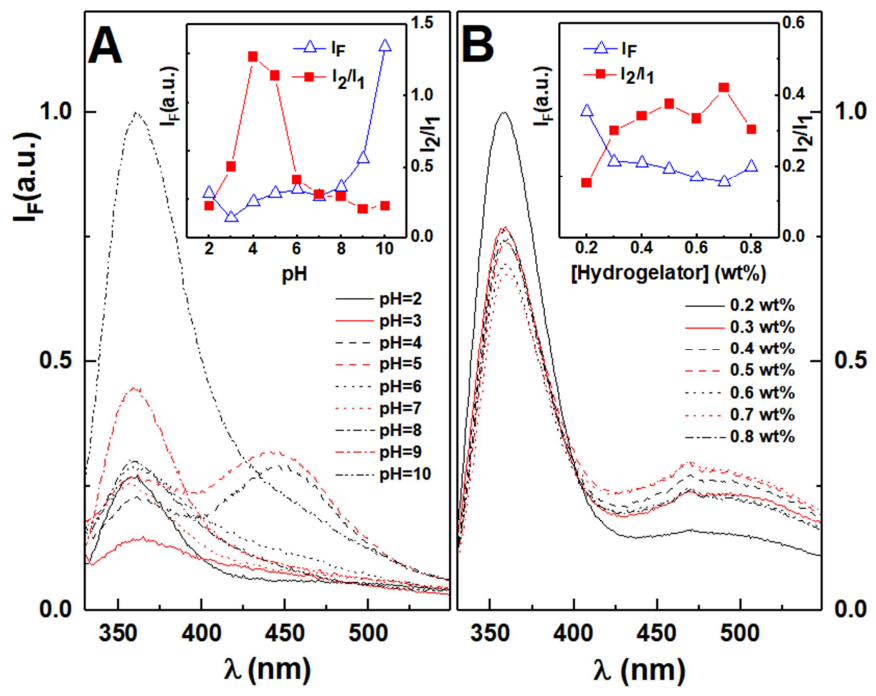

Figure 1. Fluorescence emission spectra of $(\mathbf{A})$ the hydrogelator $\left(2 \times 10^{-6} \mathrm{M}\right)$ in the $\mathrm{pH}$ range 2-10 $\left(\lambda_{\text {exc }}=290 \mathrm{~nm}\right)$ and $(B)$ in the concentration range 0.2-0.8 wt\% at $\mathrm{pH}=6\left(\lambda_{\text {exc }}=290 \mathrm{~nm}\right)$. Insets: Maximum fluorescence intensity $\left(I_{F}\right)$ variation within the $\mathrm{pH}$ range and intensity ratio $\mathrm{I}_{2} / \mathrm{I}_{1}$ of naproxen aggregate $\left(I_{2}, 450 \mathrm{~nm}\right)$ and monomer band $\left(I_{1}, 360 \mathrm{~nm}\right)$. 
Thus, the behaviour of the aggregate-to-monomer intensity ratio suggests that the carboxylic acid $\mathrm{p} K_{a}$ must be close to this $\mathrm{pH}$, which has been previously observed for other dehydropeptide-based hydrogelators containing naproxen. ${ }^{36}$

The hydrogelator critical gelation concentration (CGC) assay was carried out in $\mathrm{pH}=6$ buffer, considering that it is close to the physiological $\mathrm{pH}$, while ensuring the hydrogelator presents more $\mathrm{Npx}$ aggregates than at $\mathrm{pH}=7$. This is an advantage, considering changes in aggregation when the hydrogel gets in contact with more acidic environments, such as the tumour region. The increased concentration used in this assay (comparatively to the $\mathrm{pH}$ assay) shows no wavelength shifts of the monomer band, while the aggregate band is redshifted to $490 \mathrm{~nm}$ (Figure 1B). Moreover, in this concentration range, it is observed an incremental quenching of the monomer fluorescence emission associated with a rising aggregate band, where the minimum is attained at $0.7 \mathrm{wt} \%$. The aggregate/monomer intensity ratio notably increases from $0.2 \mathrm{wt} \%$ to $0.3 \mathrm{wt} \%$, with no changes on the monomer emission wavelength, suggesting that gelation is attained close to this concentration, where the presence of aggregates is higher. Nevertheless, a stable hydrogel could be attained at 0.1 wt\%, which was gelated by dilution in a $\mathrm{NaOH}(1 \mathrm{M})$ aqueous solution ( $2 \mathrm{v} / \mathrm{v} \%$ ) followed by $\mathrm{pH}$ decrease with glucono- $\delta$-lactone (GdL, 0.4 wt\%).

The critical aggregation concentration was studied in the nanomolar range (Figure S3B in Supplementary Information). An enhancement of monomer fluorescence emission band is observed until a maximum at $0.025 \mathrm{nM}$. Hereby, the maximum fluorescence emission is associated with the concentration at which self-assembly/aggregation takes place at room temperature, without applying any stimulus. The low aggregation concentration might be associated with the high predicted $\log P$ value of 4.12 .

\section{Molecular docking studies of hydrogelator Npx-L-Met-Z- $\triangle$ Phe-OH}

Considering the anti-inflammatory property of the naproxen moiety, that might inhibit unwanted side effects by the immune system upon administration of the gel, the specificity and selectivity of the hydrogelator 1 towards cyclooxygenase 2 (COX-2) and cyclooxygenase 1 (COX-1) was computationally evaluated. Direct docking of the crystallographic naproxen to evaluate the reliability of the defined protocol was previously reported and a root mean square deviation of $0.61 \AA$ was calculated. ${ }^{59}$ The direct docking of the crystallographic flurbiprofen in COX-1 (Figure $\mathrm{S} 4$ in Supplementary Information) attained a RMSD of $0.765 \AA$, thus making the defined protocol suitable.

Cross docking between the hydrogelator and cyclooxygenases followed the same procedure applied to naproxen and flurbiprofen direct docking, to evaluate the binding affinity for the active site and which parameters influence the hydrogelator stabilization in the binding pocket. The stabilizing hydrogen bonding interactions and distances to the $\mathrm{Tyr}^{355}$ hydroxyl group and $\mathrm{Arg}^{120}$ guanidinium moiety ${ }^{60}$ are represented in Figure 2. Moreover, the common hydrophobic residues $\mathrm{Leu}^{352}, \mathrm{Val}^{349}, \mathrm{Ala}^{527}$, and cyclooxygenase 2 $\left(\mathrm{Val}^{523}\right)$ and $1\left(\operatorname{Trp}^{387}\right.$, Leu $\left.{ }^{531}, \mathrm{Ser}^{530}\right)$ specific ones are also represented, due to their role in the stabilization of the crystallographic ligands (naproxen and flurbiprofen) through hydrophobic interactions. ${ }^{60}$

The obtained lowest estimated free energies of binding, final intermolecular energy contribution in the binding pocket and the internal energy (both in bound and unbound state) that result from the AutoDock calculations are presented in Table 1.

Table 1. AutoDock results in COX-2 and COX-1 for the hydrogelator compared to naproxen in COX-2 and flurbiprofen in COX-1.

\begin{tabular}{|c|c|c|c|c|}
\hline Energy (kcal/mol) & Naproxen (COX-2) ${ }^{59}$ & Flurbiprofen (COX-1) & cox-2 & cox-1 \\
\hline Estimated free energy of binding & -8.25 & -9.10 & -7.43 & -6.52 \\
\hline Final intermolecular energy & -9.15 & -10.00 & -11.01 & -10.10 \\
\hline Final total internal energy & -0.31 & -0.38 & -3.26 & -1.24 \\
\hline Loss of torsional free energy & +0.89 & +0.89 & +3.58 & +3.58 \\
\hline Unbound system energy & -0.31 & -0.38 & -3.26 & -1.24 \\
\hline
\end{tabular}
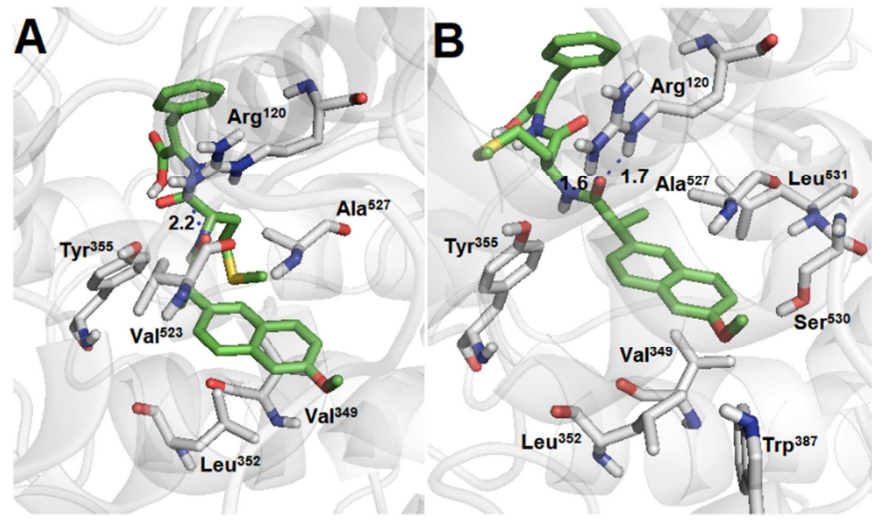

Figure 2. Representation of the best docking poses and polar interactions distances $(\AA \AA)$ for the hydrogelator in (A) COX-2 and (B) COX-1 binding pocket obtained from PyMOL software.
The hydrogelator shows a lower free energy of binding towards the cyclooxygenases than the crystallographic ligands (naproxen and flurbiprofen). The greatest contribution is associated with the favourable intermolecular interactions stablished between the binding pocket and the hydrogelator.

The methionine side chain affects the orientation of naproxen moiety in the binding pocket. Thus, the hydrogelator lower affinity than the crystallographic ligands can be accounted for the higher loss of torsional free energy parameters.

The peptide backbone seems to have introduced a slight selectivity towards COX-2. Nevertheless, the results suggest that an antiinflammatory activity through interaction with cyclooxygenases is possible, though at a lesser extent than naproxen and flurbiprofen.

Cytotoxicity assay. The effect of Npx-L-Met-Z- $\triangle$ Phe-OH hydrogelator (1) on the viability of rat macrophages (RAW 264.7 
cells) was tested. The results show that the hydrogelator did not show any statistically significant cytotoxic effects on RAW cells up to $100 \mu \mathrm{M}$ (Figure 3).

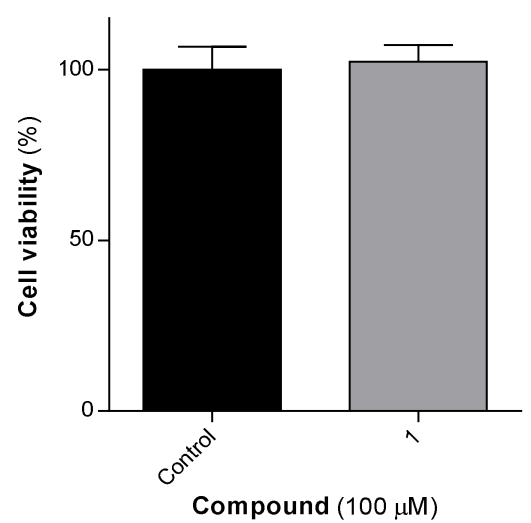

Figure 3. Cell viability in the presence of Npx-L-Met-Z- $\Delta$ Phe-OH for 24 hours (three independent experiments performed in triplicate).

\section{Synthesis of the magnetic/plasmonic nanoparticles}

The core/shell magnetic/plasmonic nanoparticles were synthesised based on the direct deposition of gold onto the manganese ferrite. $^{61}$ The gold-decorated nanoparticles were synthesised through $\mathrm{CDI}$ coupling of the nanoparticle surface $\mathrm{OH}^{-}$groups to the MPA-functionalized gold nanoparticles carboxylic moiety. The obtained UV-visible absorption spectra are represented in Figure 4. From the Tauc plot (inset of Figure $4 A$ ), the optical band gap $\left(E_{g}\right)$ of the manganese ferrite nanoparticles was determined and a linear relation was obtained for an indirect semiconductor with a band gap of $1.06 \mathrm{eV}$, which is similar to the previous reported values of $1.08 \mathrm{eV}^{37}$ and $0.98 \mathrm{eV}^{62}$ The gold shell resulted in the appearance of a wide plasmon band with maximum between 542 and $560 \mathrm{~nm}$ (Figure 4B), with a small shoulder near $850 \mathrm{~nm}$.

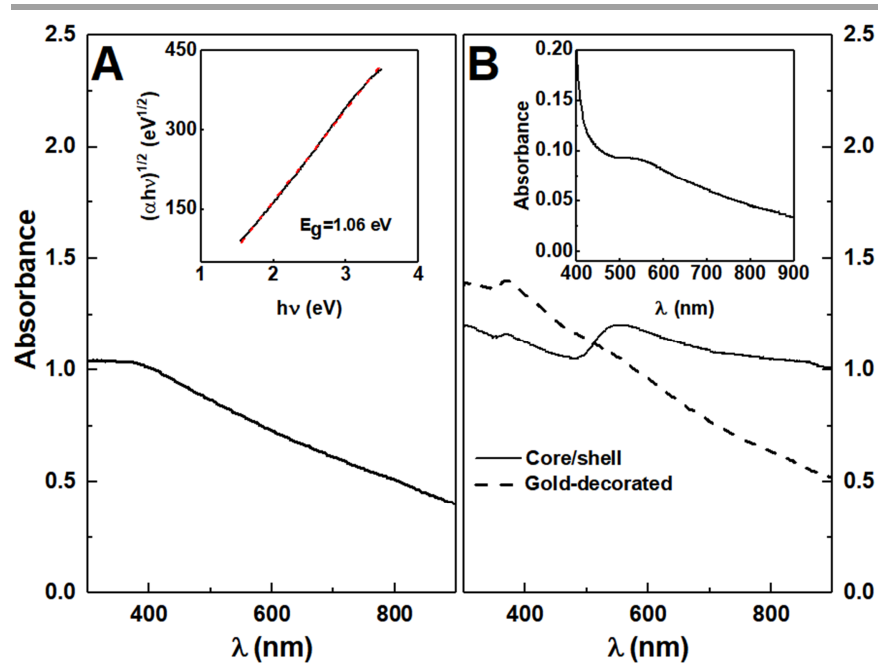

Figure 4. Absorption spectra of (A) $\mathrm{MnFe}_{2} \mathrm{O}_{4}$ nanoparticles and (B) Core/shell $\mathrm{MnFe}_{2} \mathrm{O}_{4}$ /gold and gold-decorated $\mathrm{MnFe}_{2} \mathrm{O}_{4}$ nanoparticles. Inset: (left) Tauc plot of $\mathrm{MnFe}_{2} \mathrm{O}_{4}$ nanoparticles and (right) Absorption spectrum of gold nanoparticles.
An universal scaling exponential decay of $\Delta \lambda / \lambda_{0}$ with the ratio between shell thickness and magnetite $\left(\mathrm{Fe}_{3} \mathrm{O}_{4}\right)$ core radius was proposed, ${ }^{61,63}$ where $\Delta \lambda=\lambda-\lambda_{0}, \lambda$ being the LSPR wavelength of the core-shell nanoparticle and $\lambda_{0}$ the LSPR position of the corresponding gold core. Considering cores of $9 \mathrm{~nm}, 21 \mathrm{~nm}$ and $48 \mathrm{~nm}$, the corresponding gold nanoparticles in water exhibit LSPR bands at $517 \mathrm{~nm}, 521 \mathrm{~nm}$ and $533 \mathrm{~nm}$, respectively. ${ }^{64}$ Taking into account that the medium at the surface of prepared core-shell nanoparticles is expected to be composed by glycerol, for which the refraction index (1.47) is higher than that of water, a $\sim 20 \mathrm{~nm}$ red shift in the LSPR band is expected for spherical gold nanoparticles with $40 \mathrm{~nm}$ size, ${ }^{65}$ with the magnitude of the shift decreasing with lowering particle size. Therefore, the LSPR of the considered gold cores are predicted to be at $<537 \mathrm{~nm},<541 \mathrm{~nm}$ and $>553 \mathrm{~nm}$. The LSPR of core-shell nanoparticles would then be, respectively, $<758 \mathrm{~nm},<853 \mathrm{~nm}$ and $>916 \mathrm{~nm}$, for a $1 \mathrm{~nm}$ shell and $<538 \mathrm{~nm}$, $<579 \mathrm{~nm}$ and > $691 \mathrm{~nm}$ for a $10 \mathrm{~nm}$ shell. However, the red-shifted plasmon band arising from coupling of the gold shell with the core medium was found to be damped when the core imaginary refractive index increases, ${ }^{63}$ remaining an anti-coupled mode slightly deviated to the blue. $\mathrm{MnFe}_{2} \mathrm{O}_{4}$ is expected to have a higher imaginary component of the refractive index than magnetite, as the indirect band gap is much lower than that of $\mathrm{Fe}_{3} \mathrm{O}_{4}$, which has been reported to be $2.12 \mathrm{eV}^{66}$ Thus, the obtained absorption spectrum of the Au@MnFe${ }_{2} \mathrm{O}_{4}$ nanoparticles is compatible with near $50 \mathrm{~nm}$ core and $1 \mathrm{~nm}$ gold shell, with the anti-coupled mode appearing below $553 \mathrm{~nm}$, or, considering that the coupled mode is not damped, a core near $20 \mathrm{~nm}$ and gold shell above $10 \mathrm{~nm}$.

The prepared spherical gold nanoparticles have its characteristic surface plasmon resonance band centred around $530 \mathrm{~nm}$, which can be associated to nanoparticles with a size in the range $5-10 \mathrm{~nm}$, comparing to gold nanoparticles capped with thiol ligands published in other works. ${ }^{39,67}$ Moreover, the colour shown upon nanoparticle precipitation is not associated with aggregation of nanoparticles, as no absorption band around $700 \mathrm{~nm}$ is observed. ${ }^{39}$ This means that the capping layer is sufficiently long to avoid coupling between gold nanoparticles. After coupling with manganese ferrite nanoparticles, the contribution from the gold nanoparticles plasmon band is observed around $550 \mathrm{~nm}$. This shift indicates that gold nanoparticles have been effectively bonded to manganese ferrite nanoparticles.

X-Ray Diffraction. The X-ray diffraction (XRD) pattern of manganese ferrite nanoparticles presents well-defined peaks (Figure 5), suggesting a structure of crystalline nature, even without calcination and after addition of the gold shell, as previously reported. ${ }^{37,38}$ The gold-decorated manganese ferrite nanoparticles show a neat amorphous contribution associated with the organic layer of the MPA-capped gold nanoparticles (Figure 5C).

Nevertheless, diffraction peaks of gold (Figure $5 B, C$ ) are observed at $2 \theta=38.1^{\circ}(111), 44.4^{\circ}(200), 64.6^{\circ}(220), 77.6^{\circ}(311)$ and $81.8^{\circ}$ (2 2 2), corresponding to CIF 9013035 (space group $\mathrm{Fm}-3 \mathrm{~m}$ ). Diffraction peaks of the $\mathrm{MnFe}_{2} \mathrm{O}_{4}$ crystalline structure (Figure 5A) are observed at $2 \theta=29.7^{\circ}(220), 34.9^{\circ}$ (3 11 1), $36.5^{\circ}$ (2 22 2), $42.5^{\circ}$

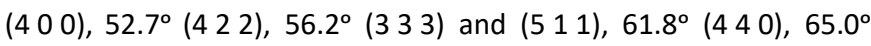
(5 3 1), $70.1^{\circ}$ (6 2 0), $73.1^{\circ}$ (5 33 3), $74.0^{\circ}(622), 78^{\circ}(444), 85.6^{\circ}$ 
(6 4 2), 88.5 (7 3 1) and (5 5 3), corresponding to CIF file 23000618 (space group Fd-3m:2).
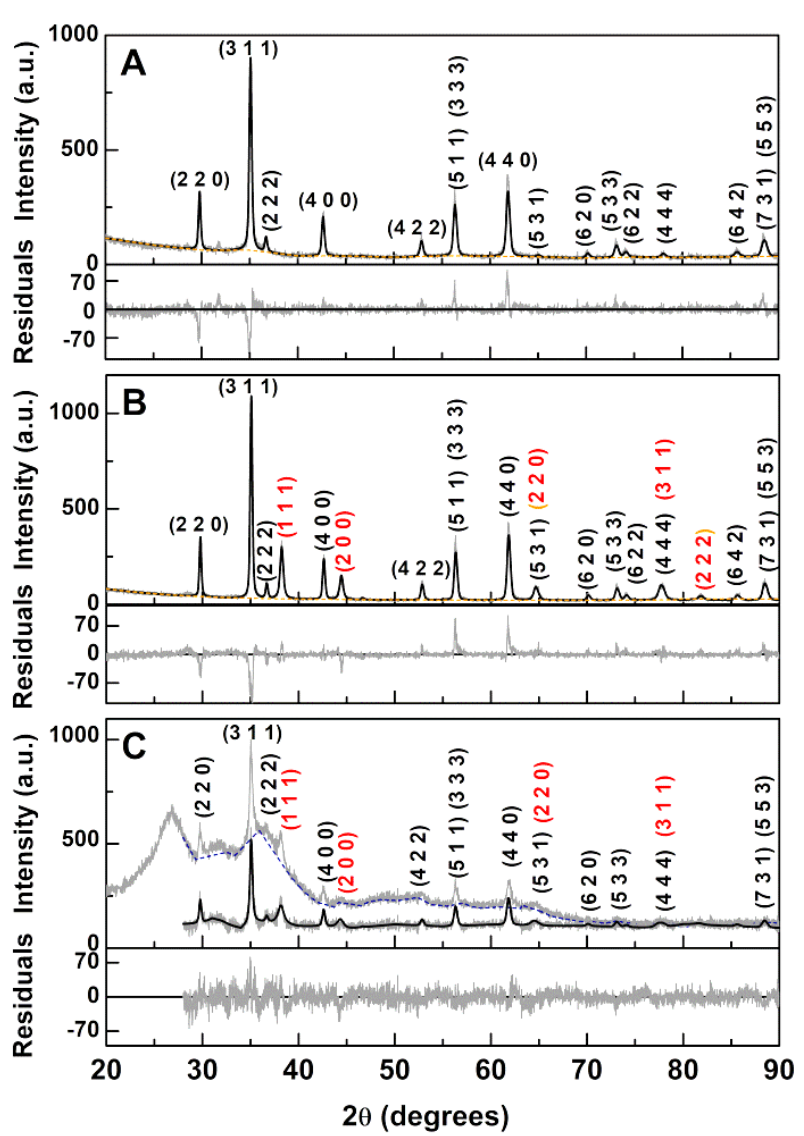

Figure 5. X-ray diffraction pattern of (A) manganese ferrite nanoparticles; (B) core/shell manganese ferrite/gold nanoparticles; (C) manganese ferrite decorated with gold nanoparticles. Gray lines: experimental patterns; black lines: fitted patterns. Background $(A, B)$ and amorphous background $(C)$ : short-dashed lines. Miller indices: Black: manganese ferrite; Red: gold.

Rietveld analysis was performed using FullProf software suite ${ }^{68}$ (details in Supplementary Information). The calculated parameters and phase sizes are presented in Table 2.

The manganese ferrite phases have an average size of approximately $34.8 \mathrm{~nm}$, that is found to increase to $48.8 \mathrm{~nm}$ upon the thermal treatment in glycerol that leads to formation of the gold shell. ${ }^{38}$ The gold phase in the core/shell nanoparticles is estimated to be $22.6 \mathrm{~nm}$, resulting in $94 \mathrm{~nm}$ overall particle size. But several studies report the absence of core diffraction peaks when the gold shell has a thickness higher than $2 \mathrm{~nm},{ }^{69,70}$ or a huge intensity decrease with gold shell up to $8.5 \mathrm{~nm} .{ }^{71}$ In the latter case, a $13 \mathrm{~nm}$ core is observed to have a broader diffraction peak than a $2.5 \mathrm{~nm}$ shell. On the other hand, gold shell diffraction peaks seem to have distinct broadening effects, as for $10 \mathrm{~nm}$ magnetite core coated with a $2 \mathrm{~nm}$ gold shell the diffraction peak widths were identical (where its dimensions were obtained from TEM measurements). ${ }^{69}$ Thus, in the obtained core/shell nanoparticles, either the gold shell is incomplete, or a thin shell is present. The obtained gold weight percentage is only $7.1 \%$. If the sizes obtained from the Rietveld analysis were the correct ones, and even considering that the size of gold phase corresponds to the double of the thickness (a given X-Ray when interacting with a core-shell nanoparticle encounters gold phase two times), a $90 \%$ value would be expected ( $71.4 \mathrm{~nm}$ overall size). This means that only $8 \%$ of the $\mathrm{MnFe}_{2} \mathrm{O}_{4}$ nanoparticles would have been coated. Considering a $1 \mathrm{~nm}$ shell thickness $(50.8 \mathrm{~nm}$ overall size), then $33 \%$ weight percentage is expected, with a corresponding more reasonable $21 \%$ value for the coating efficiency.

For the gold-decorated nanoparticles and considering again that the gold phase size given by the Rietveld analysis corresponds to the double of the gold nanoparticles size, the obtained gold weight percentage indicates that the average number of gold nanoparticles per $\mathrm{MnFe}_{2} \mathrm{O}_{4}$ is 16.8 . This value would be 2.1 if the gold phase size corresponds to the gold nanoparticles size.

Table 2. X-ray diffraction Rietveld refinement calculated parameters $R_{f}$ and $\chi^{2}$, phase sizes and percentages. $\mathrm{MnFe}_{2} \mathrm{O}_{4} / \mathrm{Au}$ : core/shell nanoparticles; $\mathrm{Au} @ \mathrm{MnFe}_{2} \mathrm{O}_{4}$ : gold-decorated $\mathrm{MnFe}_{2} \mathrm{O}_{4}$ nanoparticles.

\begin{tabular}{|c|c|c|c|c|c|c|}
\hline \multirow[t]{2}{*}{ Nanoparticles } & \multicolumn{2}{|c|}{$\begin{array}{c}\text { Intensity } \\
\text { percentages }\end{array}$} & \multicolumn{2}{|c|}{$\begin{array}{l}\text { Phase size }(\mathrm{nm}) \\
\text { Lattice Constant }(\AA)\end{array}$} & \multicolumn{2}{|c|}{$\begin{array}{c}\text { Quality } \\
\text { Parameters } \\
\mathrm{MnFe}_{2} \mathrm{O}_{4} \mid \mathrm{Au}\end{array}$} \\
\hline & $\mathrm{MnFe}_{2} \mathrm{O}_{4}$ & $\mathrm{Au}$ & $\mathrm{MnFe}_{2} \mathrm{O}_{4}$ & $\mathrm{Au}$ & $\mathbf{R}_{\mathrm{f}}$ & $x^{2}$ \\
\hline $\mathrm{MnFe}_{2} \mathrm{O}_{4}$ & 100 & --- & $\begin{array}{c}34.8 \\
8.481\end{array}$ & --- & 7.88 & 1.03 \\
\hline $\mathrm{MnFe}_{2} \mathrm{O}_{4} / \mathrm{Au}$ & 92.9 & 7.1 & $\begin{array}{c}48.8 \\
8.483\end{array}$ & $\begin{array}{c}22.6 \\
4.076\end{array}$ & $7.01 \mid 3.52$ & 0.93 \\
\hline $\mathrm{Au} @ \mathrm{MnFe}_{2} \mathrm{O}_{4}$ & 88.9 & 11.1 & $\begin{array}{c}34.8\left(^{*}\right) \\
8.486\end{array}$ & $\begin{array}{c}8.64 \\
4.085\end{array}$ & $9.32 \mid 6.00$ & 1.53 \\
\hline
\end{tabular}

$(*)$ fixed value

\section{Development of magnetogels}

The structure of the hydrogel is emphasized in Figure 6A and 6B, where it can be observed that the hydrogel matrix comprises a fibrous structure, with fibres exhibiting average cross-section of $16.6 \pm 2.4 \mathrm{~nm}$, the thinnest being $7 \mathrm{~nm}$ and the thickest $21 \mathrm{~nm}$. The fibres attain diameters larger than $2 \mu \mathrm{m}$.

Magnetogels were developed by mixing magnetic nanoparticles with the hydrogelator solution at $\mathrm{pH}=10$ and left incubating at room temperature for 4 hours (figure 6C). During the self-assembly process trigger, several phenomena will be competing, mainly the sedimentation and aggregation of nanoparticles, the formation of fibrils and coating of the nanoparticles. Hereby, the magnetogels were previously evaluated at 10, 20 and $30 \mathrm{~m} / \mathrm{m} \%$ concentration of nanoparticles for 0.3 wt\% of hydrogelator, as no magnetogel was possible to be formed at a lower hydrogelator concentration. A homogenous dispersed magnetogel was obtained for $10 \mathrm{~m} / \mathrm{m} \%$ (Figure 6D, 6E and $6 \mathrm{~F}$ ) owing to the rapid gelation, while for $20 \mathrm{~m} / \mathrm{m} \%$ core/shell nanoparticles a gradient was obtained, and at $30 \mathrm{~m} / \mathrm{m} \%$ both nanoparticles sedimented. 

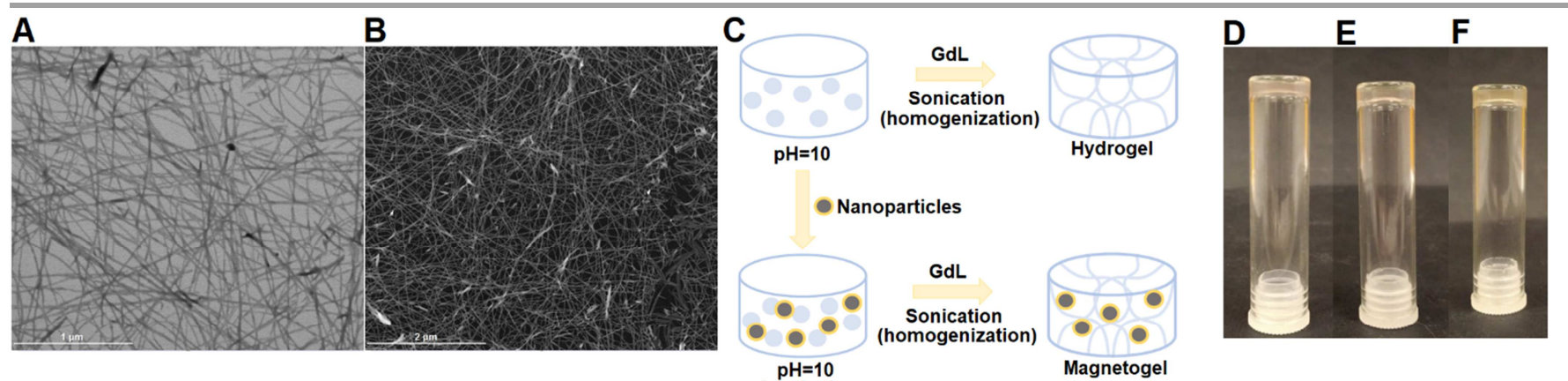

Figure 6. (A) STEM image of hydrogel in bright field (scale bar: $1 \mu \mathrm{m}$ ). (B) STEM image of hydrogel in dark field (scale bar: $2 \mu \mathrm{m}$ ). (C) Scheme of the gelation strategy of hydrogel and magnetogel. (D-F) Images of the glass vial inversion test of the magnetogel containing (D) core/shell nanoparticles $10 \mathrm{~m} / \mathrm{m} \%$; (E) gold-decorated nanoparticles at $10 \mathrm{~m} / \mathrm{m} \%$ and $(\mathbf{F})$ at $20 \mathrm{~m} / \mathrm{m} \%$. The magnetogels show a homogeneous dispersion and no visible deposition.

Rheological properties. The mechanical spectra of equilibrated Npx-L-Met-Z- $\Delta$ Phe-OH hydrogel and magnetogels $(10,20$ and 30 $\mathrm{m} / \mathrm{m} \%)$ are shown in Figure 7. The rheological data measured for magnetogels formulated with $10 \mathrm{~m} / \mathrm{m} \%$ nanoparticles underline the effects of the nanoparticles chemical modification on hydrogel elasticity. The addition of gold-decorated manganese ferrite nanoparticles results in the reinforcement of the hydrogel elasticity, as $\mathrm{G}^{\prime}$ is five times larger (Figure $7 \mathrm{~A}$ ). In contrast to this, the addition of $10 \mathrm{~m} / \mathrm{m} \%$ core/shell nanoparticles leads to a significant reduction (10 times) of the gel elasticity (Figure 7B).

Depending on the function and location of the tissue, the elastic modulus of native tissues ranges from $0.1 \mathrm{kPa}$ (brain) to $100 \mathrm{kPa}$ (cartilage). ${ }^{72}$ The developed hydrogel and magnetogels elastic modulus fall in the range $1 \mathrm{kPa}$ to $100 \mathrm{kPa}$, which covers soft tissues such as skin, pancreas, spleen, glands and muscles. ${ }^{72}$ Therefore, from a mechanical perspective, the hydrogel and magnetogels elastic properties seem to be promising for biomedical applications.
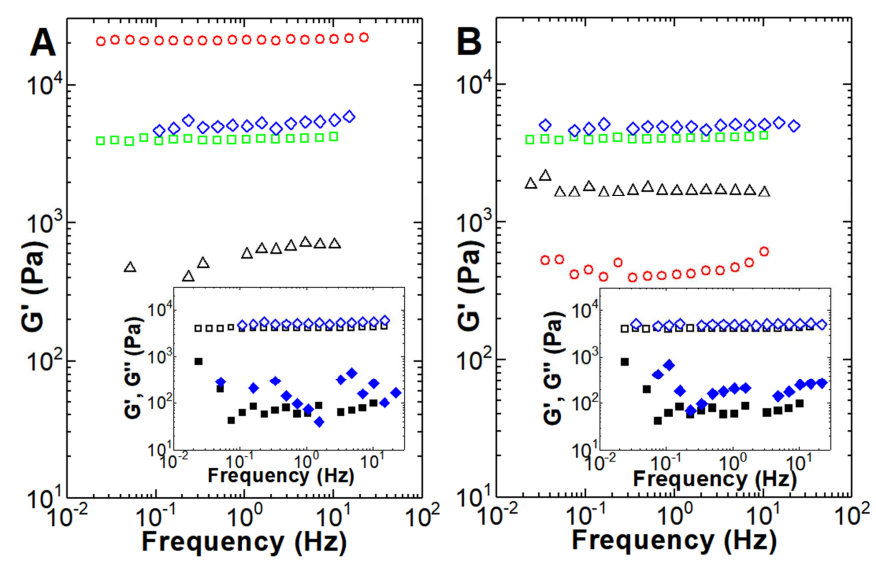

Figure 7. Frequency dependence of the shear elastic modulus $\mathrm{G}^{\prime}$ for hydrogels formulated with $0 \mathrm{~m} / \mathrm{m} \%$ (squares), $10 \mathrm{~m} / \mathrm{m} \%$ (circles), $20 \mathrm{~m} / \mathrm{m} \%$ (triangles) and $30 \mathrm{~m} / \mathrm{m} \%$ (diamonds) of gold-decorated nanoparticles (A) or core/shell nanoparticles (B). Insets: mechanical spectra ( $G^{\prime}$, empty symbols; $\mathrm{G}^{\prime \prime}$, solid symbols) of the hydrogel (squares) and of the magnetogels formulated with $30 \mathrm{~m} / \mathrm{m} \%$ nanoparticles (diamonds).
Relatively to previous reported hydrogels with similar chemical nature and prepared at similar concentration, the methionine residue hydrophobic collapse seems to have reinforced the elasticity of the hydrogel ( $4 \mathrm{kPa}$ at $0.3 \mathrm{wt} \%$ ) compared to hydrogels containing phenylalanine (Npx-L-Phe-Z- $\triangle \mathrm{Phe}-\mathrm{OH}, 1.7 \mathrm{kPa}$ at 0.4 $w t \%)$, valine (Npx-L-Val-Z- $\triangle \mathrm{Phe}-\mathrm{OH}, 0.7 \mathrm{kPa}$ at $0.6 \mathrm{wt} \%$ ) or alanine (Npx-L-Ala-Z- $\triangle \mathrm{Phe}-\mathrm{OH}, 0.8 \mathrm{kPa}$ at $0.8 \mathrm{wt} \%$ ), being similar to the tryptophan containing hydrogel (Npx-L-Trp-Z- $\triangle \mathrm{Phe}-\mathrm{OH}, 4 \mathrm{kPa}$ at 0.4 wt\%). ${ }^{34,36}$ Thus, a long hydrophobic residue close to the $\mathrm{N}$-terminal aromatic moiety is expected to favour a higher elasticity. The addition of more nanoparticles changes the picture, since the viscoelastic features of the hydrogel are progressively recovered. As a result, adding $30 \mathrm{~m} / \mathrm{m} \%$ nanoparticles does not bring any significant viscoelastic change to the hydrogel (mechanical spectra of gels at $20 \mathrm{~m} / \mathrm{m} \%$ are presented in Supplementary Information).

Nonlinear viscoelastic data were recently shown to be efficient in revealing the hierarchical structures of gelatin gels. ${ }^{73}$ Therefore, large amplitude oscillatory shear strain sweeps (LAOSS) were performed in an attempt to assess possible structural differences in gels prepared with the two types of particles. The strain dependence of the ratio $G^{\prime} / G_{0}\left(G_{0}\right.$ being the value of the shear modulus $\mathrm{G}^{\prime}$ measured at $1 \mathrm{~Hz}$ in the linear viscoelastic regime) is presented in Figure 8.

Overall, the strain dependence of the ratio $G^{\prime} / G_{0}$ indicates a strain hardening behavior for all samples but the gel formulated with $20 \mathrm{~m} / \mathrm{m} \%$ gold-decorated manganese ferrite nanoparticles. Strain hardening, characterized by an increase in $\mathrm{G}^{\prime}$ with the strain, is the nonlinear mechanical signature of a wide range of gels with structures ranging from networks of semi-flexible filaments to networks of strongly linked fractal flocs of colloidal particles. ${ }^{74-76}$

Magnetogels formulated with the gold-decorated manganese ferrite nanoparticles are more sensitive to shear deformation, since the critical strain beyond which the strain hardening kicks in is smaller ( $0.2 \%$ against $0.4 \%)$. In addition, these magnetogels exhibit a more intense hardening, as the maxima in $G^{\prime} / G_{0}$ are larger than those measured for hydrogels filled with bare core/shell nanoparticles. 

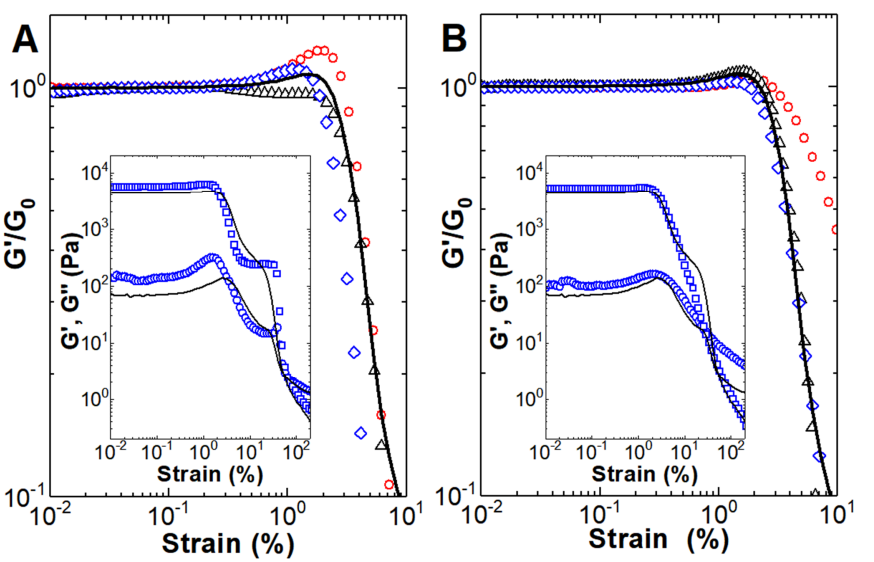

Figure 8. Strain dependence of the shear elastic modulus $G^{\prime}$ scaled by its value $\left(G_{0}\right)$ measured in the linear regime for hydrogels formulated with golddecorated nanoparticles (A) and with core/shell nanoparticles (B): $0 \mathrm{~m} / \mathrm{m} \%$ (lines), $10 \mathrm{~m} / \mathrm{m} \%$ (circles), $20 \mathrm{~m} / \mathrm{m} \%$ (triangles) and $30 \mathrm{~m} / \mathrm{m} \%$ (diamonds). Inset: Strain dependence of the elastic $\left(G^{\prime}\right.$, squares) and loss $\left(G^{\prime \prime}\right.$, circles) moduli for hydrogels formulated with $0 \mathrm{~m} / \mathrm{m} \%$ (lines) and $30 \mathrm{~m} / \mathrm{m} \%$ (symbols) of nanoparticles.

All gels showed the Payne effect, as can be seen in the inset of Figure 8 , for the hydrogel and magnetogels doped with $30 \mathrm{~m} / \mathrm{m} \%$ nanoparticles. The Payne effect has been reported for a large variety of viscoelastic matrices reinforced by solid fillers and is characterized by a local maximum in $\mathrm{G}^{\prime \prime}$, which is concomitant with a significant decrease of $G^{\prime}$ with the increasing strain. Such effect can be associated with the breakage and recovery of weak interaction bonds linking adjacent filler clusters, the aggregation/disaggregation of nanoparticles, or the molecular disentanglement. ${ }^{77}$ Nevertheless, data displayed in Figure 8 indicate that the nonlinear viscoelastic response of samples is sensitive to differences in particle-particle and particle-hydrogelator interactions.

Magnetogels characterization. The addition of core/shell nanoparticles prior to solution $\mathrm{pH}$ increase with $\mathrm{NaOH}$ resulted in the formation of irreversible aggregates, which was confirmed by UV-visible absorption spectroscopy (Figure S6 in Supplementary Information). Therefore, core/shell nanoparticles could only be added to the solution after $\mathrm{pH}$ increase, while the gold-decorated ones did not required this specific procedure.

The intrinsic fluorescence of hydrogelator naproxen moiety allows following the incorporation of magnetic nanoparticles into the hydrogel through changes on the photophysical behaviour. The fluorescence spectra show a maximum emission at $360 \mathrm{~nm}$, associated with the monomer state of the naproxen moiety, and the aggregates band around $450 \mathrm{~nm}^{36}$ (Figure 9A). The magnetic nanoparticles introduce several photophysical effects, mainly the fluorescence quenching. Such effect can be a result from the electronic energy transfer to the nanoparticles owing to its wide absorption spectrum, but also due to the heavy atom effect, where the presence of heavy atoms enhances the spin-orbit interaction that favours the intersystem crossing. ${ }^{47}$

However, more effects are included due to the localized surface plasmon of the gold shell. The near-field enhancement close to the nanoparticles will increase absorption and fluorescence, where the latter is enhanced owing to the coupling of the lowest order plasmonic modes (electric dipolar mode) to the far-field. ${ }^{78-80}$
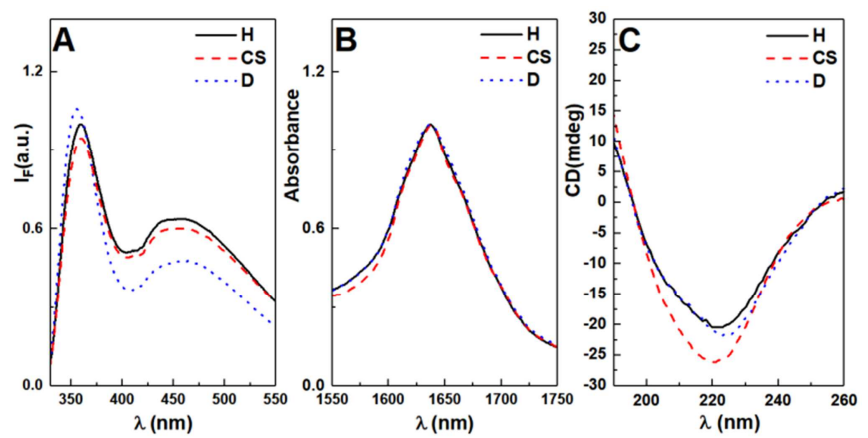

Figure 9. (A) Fluorescence emission spectra of hydrogel $(\mathrm{H})$ and magnetogels with core/shell (CS) and gold-decorated (D) nanoparticles, $10 \mathrm{~m} / \mathrm{m} \%$. (B) Hydrogel and magnetogels amide I infrared absorption spectra. (C) Hydrogel and magnetogels circular dichroism spectra of the hydrogelator solution at 0.02 wt\%.

Nevertheless, the obtained fluorescence quenching evidences the predomination of the non-radiative mechanisms on the magnetogels. On the other hand, the introduction of gold-decorated manganese ferrite nanoparticles surface functional groups might hinder the proximity of the fibres or modify its structure, as denoted by the higher monomer-to-aggregate intensity ratio and the blueshift in monomer emission (Figure 9A).

Moreover, the naproxen moiety fluorescence anisotropy values suggest that the hydrogel $(r=0.113)$ fibrils have suffered a structural change with the introduction of the core/shell $(r=0.043)$ and gold-decorated $(r=0.035)$ nanoparticles.

Figure $9 \mathrm{~B}$ displays the FTIR absorption spectra in the amide I region (1600-1700 $\left.\mathrm{cm}^{-1}\right)$. In this region, the main contribution for infrared absorption is the $\mathrm{C}=\mathrm{O}$ stretching, which is sensible to the protein secondary structure owing to the hydrogen bonding pattern and molecular geometry. The second derivative shows a peak around $1625 \mathrm{~cm}^{-1}$ and $1639-1641 \mathrm{~cm}^{-1}$ that can be assigned to $\beta$-sheet and random coil, respectively (Figure S7 in Supplementary Information). Other peaks were identified at $1668 \mathrm{~cm}^{-1}$ and $1685 \mathrm{~cm}^{-1}$, being associated with the presence of turns and loops, while a peak at $1699 \mathrm{~cm}^{-1}$ might be a result of strong hydrogen bonds between $\beta$ sheets or $\beta$-strands aggregates. ${ }^{49}$ The major difference is that magnetogels have a decrease in intensity of the bands of ordered structures, suggesting that, in the presence of nanoparticles, the hydrogelators are more randomly oriented. Table 3 displays the percentages of secondary structures obtained for the hydrogel and magnetogels. The results show a close agreement between the amide III and second derivative curve fitting method, mainly when the percentages of ordered ( $\alpha$-helix and $\beta$-sheet) and unordered (random coil and turns) structures are compared. Overall, a reduction of ordered secondary structures was obtained on the magnetogels when compared to the hydrogels, which is accompanied by an increase of unordered structures. Hereby, a higher percentage of unordered structures might reduce the compactness of the well-ordered self-assembled structures, owing to the lack of ordered hydrogen bonding, which not only might induce thicker fibres than in the hydrogel, but also a less stable matrix. 
Table 3. Percentage of secondary structures according to the ranges reported in literature, ${ }^{48-52}$ obtained through curve-fitting of the amide I band (Al), amide I second derivative (ASD) and amide III band (AIII). Legend: OC: ordered structures ( $\alpha$-helix and $\beta$-sheet); UC: unordered structures (turn and random coil).

\begin{tabular}{|c|c|c|c|c|c|c|c|c|c|}
\hline \multirow{2}{*}{$\begin{array}{l}\text { System } \\
\text { Method }\end{array}$} & \multicolumn{3}{|c|}{$\mathrm{H}$} & \multicolumn{3}{|c|}{ CS } & \multicolumn{3}{|c|}{ D } \\
\hline & Al & ASD & AIII & Al & ASD & AIII & Al & ASD & AllI \\
\hline$\alpha$-helix & 0 & 0 & 9.8 & 0 & 0 & 13.3 & 0 & 0 & 12.8 \\
\hline$\beta$-sheet & 45.8 & 28.1 & 19.7 & 41.6 & 24.3 & 13.0 & 34.4 & 25.2 & 13.0 \\
\hline Turn & 19.9 & 17.0 & 23.0 & 9.4 & 24.5 & 33.9 & 41.2 & 16.3 & 32.0 \\
\hline Random coil & 34.3 & 54.9 & 47.4 & 49.0 & 44.2 & 39.8 & 24.4 & 58.5 & 42.2 \\
\hline OC & 45.8 & 28.1 & 29.6 & 41.6 & 26.1 & 26.3 & 34.4 & 25.2 & 25.8 \\
\hline UC & 54.2 & 71.9 & 70.4 & 58.4 & 73.9 & 73.7 & 65.6 & 74.8 & 74.2 \\
\hline$x^{2}$ & $4 \times 10^{-7}$ & $1 \times 10^{-11}$ & $3 \times 10^{-8}$ & $4 \times 10^{-8}$ & $3 \times 10^{-11}$ & $7 \times 10^{-10}$ & $2 \times 10^{-7}$ & $6 \times 10^{-12}$ & $1 \times 10^{-10}$ \\
\hline
\end{tabular}

A molecule is optically active in circular dichroism (CD) if the chromophore contains an asymmetric (chiral) environment or display an induced chirality owing to three-dimensional bonding structures. ${ }^{81-83}$ Hereby, the spectrum corresponds to the sum of its conformational elements. ${ }^{83}$ The $\beta$-sheet structures are characterized by a sharp positive band in the region 195-210 nm and a broad negative band in the region 215-230 nm, while $\alpha$-helices display negative ellipticity at $222 \mathrm{~nm}$ and $208 \mathrm{~nm}$, and positive ellipticity at $193 \mathrm{~nm} .{ }^{83}$ The random coil structures have a weak positive band at $217 \mathrm{~nm}$ and a large negative near $200 \mathrm{~nm} .^{83}$ These bands are associated with the peptide bond transitions $\pi-\pi^{*}$ and $n-\pi^{*}$, which are centred around $190 \mathrm{~nm}$ and $220 \mathrm{~nm}$, respectively. ${ }^{36,84}$ The obtained $C D$ spectra of diluted hydrogelator solutions, with and without nanoparticles, are represented in Figure 9C. The solutions show similar spectral shape, where an increase of ellipticity is observed in the presence of core/shell nanoparticles and a red-shift for the magnetogel containing gold-decorated nanoparticles. Overall, the exciton couplets are evidenced by a negative cotton effect with a broad negative ellipticity centred at $220 \mathrm{~nm}$ and a slow ellipticity decrease for lower wavelengths, which suggest a predominance of $\beta$-sheets and that hydrogelator molecules establish similar intermolecular interactions in hydrogel and magnetogels. Nevertheless, the naphthalene moiety commonly shows exciton couplets between 200 and $230 \mathrm{~nm}$, besides being stronger in magnitude than the peptide transitions. ${ }^{85}$ Thus, the broad negative ellipticity arises from the sum of contributions of secondary structure and naphthalene exciton couplets.

Transmission Electron Microscopy. The transmission electron microscopy (TEM) images of the magnetic nanoparticles and magnetogels are presented in Figure 10. Core/shell manganese ferrite/gold nanoparticles show an average size of $55 \pm 14 \mathrm{~nm}$, while the gold-decorated manganese ferrite nanoparticles show diameters of $45.7 \pm 8 \mathrm{~nm}$ containing gold nanoparticles with $3.8 \pm 0.9 \mathrm{~nm}$ (size histograms of the nanoparticles in Figure S8 of Supplementary Information). The electron dispersive X-ray (EDX) spectra evidence the presence of gold in both types of nanoparticles (Figures S9 and S10 in Supplementary Information).

For the core-shell nanoparticles, subtracting (from the obtained average size) the value of $\mathrm{MnFe}_{2} \mathrm{O}_{4}$ core diameter that resulted through XRD Rietveld analysis, the shell thickness can be estimated as $3 \mathrm{~nm}$. But the defect on Au coating seen in the darker zone of Figure $10-\mathrm{A} 3$ seems to indicate a much thinner shell layer. Fast
Fourier Transform (FFT) of this figure shows bright spots that prove the existence of both $\mathrm{MnFe}_{2} \mathrm{O}_{4}$ and $\mathrm{Au}$ phases (Figure 10-A4). Specifically, these spots appear at 2.98, 2.66, 1.70, 1.58, 1.45 and $1.34 \AA$, corresponding to $\mathrm{d}$-spacing values of the $\mathrm{MnFe}_{2} \mathrm{O}_{4}$ spinel crystal structure with $8.483 \AA$ lattice constant of $3.00,2.56,1.73$, $1.63,1.43$ and $1.34 \AA$, where the (h k l) Miller indices are indicated in the figure. The Au phase originates spots at $1.45,1.08$ and $0.9 \AA$, that are assignable to $d$-spacing values of the $A u$ fcc crystal structure with $4.076 \AA$ lattice constant of $1.44,1.02$ and $0.91 \AA$. The bright spots at low spacing values $(\sim 0.4 \AA$ and $0.8 \AA$ ) and marked with a dark arrow, are found to be instrumental artifacts, as they appear in the FFT of images with different resolutions at exactly the same pixel positions (data not shown). FFT of circular spots in the lighter and darker regions of Figure 10-A3 (Figure S11 in Supplementary Information) shows that the gold spots mainly originate from the darker region.

For the gold decorated $\mathrm{MnFe}_{2} \mathrm{O}_{4}$ nanoparticles, the estimation of overall particle size was very difficult due to extended agglomeration in the TEM grid. That explains why the obtained average value of $45.7 \mathrm{~nm}$ is higher than the sum of the size of $\mathrm{MnFe}_{2} \mathrm{O}_{4}$ phase obtained from XRD Rietveld analysis $(34.8 \mathrm{~nm}$ ) with that of the $\mathrm{Au}$ nanoparticles obtained from TEM $(3.8 \mathrm{~nm})$. Nevertheless, the size of gold nanoparticles from TEM imaging is similar to half the value of the size determined from XRD for the gold phase $(7.88 / 2=3.94 \mathrm{~nm})$, confirming the hypothesis that, in average, the $\mathrm{X}$-rays at a given diffraction angle, detected two gold nanoparticles per $\mathrm{MnFe}_{2} \mathrm{O}_{4}$ core. The FFT of image in Figure 10-B3 also shows bright spots that confirm the presence of both $\mathrm{Au}$ and $\mathrm{MnFe}_{2} \mathrm{O}_{4}$ phases. Specifically, these spots appear at 4.72, 2.98, 2.54, $1.89,1.61,1.51$ and $1.42 \AA$, corresponding to d-spacing values of the $\mathrm{MnFe}_{2} \mathrm{O}_{4}$ spinel crystal structure with $8.486 \AA$ lattice constant of $4.90,3.00,2.56,1.94,1.63,1.50$ and $1.41 \AA$. For $\mathrm{Au}$, the bright spots are observed at $2.38,1.98$ and $1.42 \AA$ and are attributable to d-spacing values of the $A u f c c$ crystal structure with $4.085 \AA$ lattice constant of 2.35, 2.04 and $1.44 \AA$.

In Figures $10-\mathrm{C} 1$ and $10-\mathrm{C} 2$, it can be observed that the nanoparticles work as template surfaces for the growth of the hydrogel fibres, which results in a porous structure with an average pore size of $82.2 \pm 44.6 \mathrm{~nm}$, where the smallest pore is $20 \mathrm{~nm}$ and the largest is $223 \mathrm{~nm}$. The fibres have an average cross section of $11.2 \pm 3.6 \mathrm{~nm}$, the thinnest being $5.6 \mathrm{~nm}$ and the thickest $21 \mathrm{~nm}$. The high density of nanoparticles and fibre crosslinks suggest that the gels are highly saturated at $10 \mathrm{~m} / \mathrm{m} \%$ of nanoparticles. 

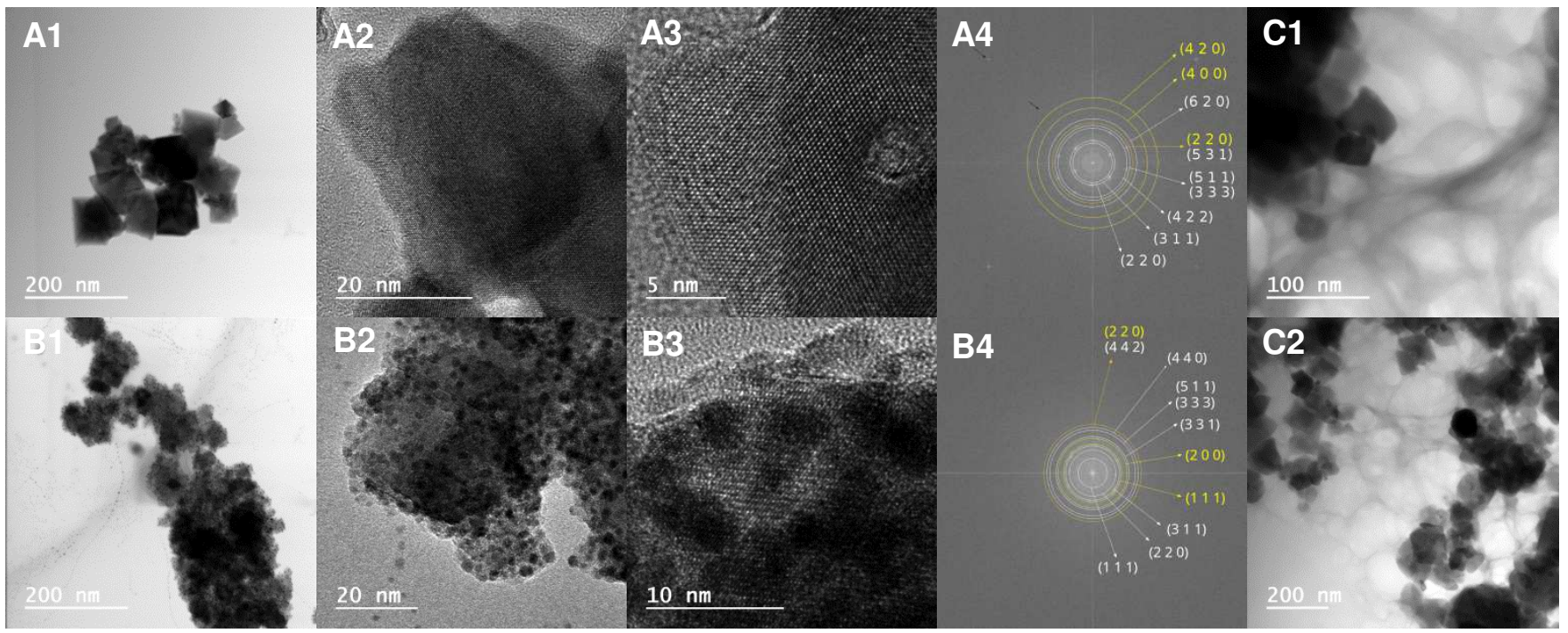

Figure 10. Transmission electron microscopy images of (A) Core/shell manganese ferrite/gold nanoparticles; (B) gold-decorated manganese ferrite nanoparticles. A4 and B4 are the Fast Fourier Transform of the respective nanoparticles, where some gold planes (yellow) and $\mathrm{MnFe}_{2} \mathrm{O}_{4}$ planes (white) were indexed. (C) TEM image of Npx-L-Met-Z- $\triangle$ Phe-OH (0.08 wt\%) magnetogel containing $10 \mathrm{~m} / \mathrm{m} \%$ core/shell manganese ferrite/gold nanoparticles (C1) and gold-decorated manganese ferrite nanoparticles (C2).

Magnetic properties. The magnetic properties can be determined from the magnetic hysteresis loop, which relates the induced magnetic moment with the applied magnetic field $(\mathrm{H})$. It can be observed that the maximum magnetization (emu/g) is higher for the core/shell nanoparticles than for the decorated ones (Figure 11). This behavior can be explained considering the high amorphous content of the decorated nanoparticles due to the organic component, as these nanoparticles are functionalized with MPA. On the other hand, the result for core/shell nanostructures is consistent with a thin gold shell, as the saturation magnetization is much higher than the previously reported for $\mathrm{MnFe}_{2} \mathrm{O}_{4}$ nanoparticles with a $\sim 5 \mathrm{~nm}$ gold shell ${ }^{38}$ (Table 4 ).

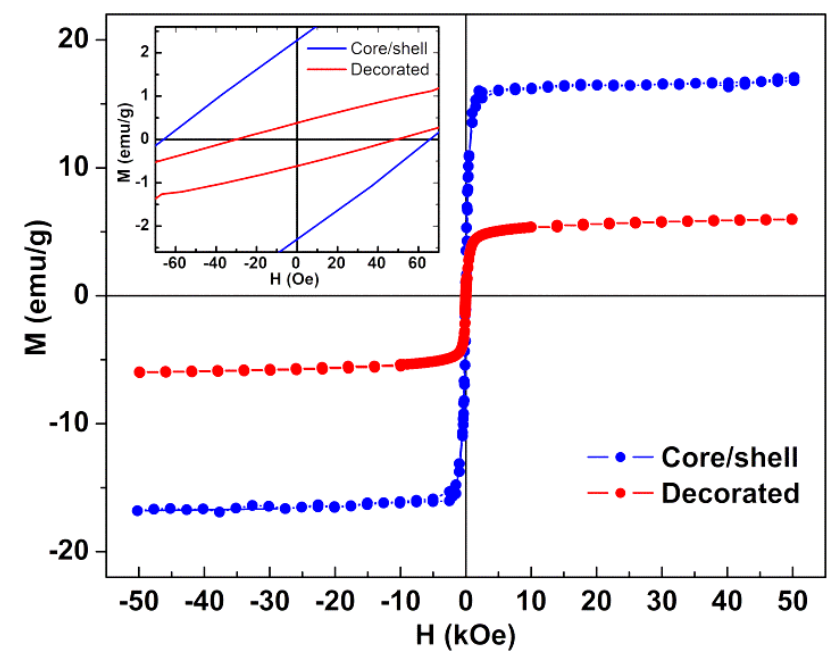

Figure 11. Magnetization hysteresis loops of manganese ferrite/gold nanoparticles measured at room temperature ( $\mathrm{T}=300 \mathrm{~K})$. Inset: Enlargement of the loops in the low field region.
Table 4. Coercive field $\left(H_{c}\right)$, saturation magnetization $\left(M_{s}\right)$, remnant magnetization $\left(M_{r}\right)$, and ratio $M_{r} / M_{s}$ for core/shell manganese ferrite/gold nanoparticles and gold-decorated manganese ferrite nanoparticles, at room temperature $(\mathrm{T}=300 \mathrm{~K})$.

\begin{tabular}{lcccc}
\hline & $\mathrm{H}_{\mathrm{c}}(\mathrm{Oe})$ & $\mathrm{M}_{\mathrm{s}}$ (emu/g) & $\mathbf{M}_{\mathrm{r}}(\mathrm{emu} / \mathrm{g})$ & $\mathbf{M}_{\mathrm{r}} / \mathbf{M}_{\mathbf{s}}$ \\
\hline Core/shell & 63.2 & 17.1 & 2.04 & 0.12 \\
Decorated & 39.1 & 6.15 & 0.52 & 0.08 \\
\hline
\end{tabular}

As evidenced from the $M_{r} / M_{s}$ ratio, which is around 0.1 for both types of nanoparticles, both nanoparticles present roughly a superparamagnetic behaviour. ${ }^{86}$

Therefore, both nanoparticles design shows promising results for magnetic targeting and hyperthermia, though the gold-shell manganese ferrite nanoparticles design represents an improvement due to the reduction of the diamagnetic contribution of gold. The presence of both plasmonic and magnetic materials with promising magnetic properties is expected to synergistically enhance both photodynamic therapy and hyperthermia (through the combined use of magnetic hyperthermia and photothermia), to allow the use of the unique modality magnetomotive photoacoustic imaging, ${ }^{61}$ and to guarantee the retention of nanoparticles in the target site (through a magnetic field gradient) to avert the reoccurrence of cancer even when the hydrogel has been degraded/absorbed.

Drug encapsulation. Curcumin, here used as model drug, is barely soluble in water, ${ }^{87}$ and its fluorescence emission will be an indicator of its presence in the hydrophobic cavities or near the fibres. The spectral overlap between the hydrogelator aromatic groups fluorescence and curcumin absorption shows that Förster resonance energy transfer (FRET) is possible, which allows following the incorporation of curcumin into the hydrogel/magnetogels (Figure S12 in Supplementary Information). 
The fluorescence emission (around $500 \mathrm{~nm}$ ) of curcumin due to FRET from the hydrogelator is overlapped by the aggregates emission band (Figure 12). However, direct excitation at $420 \mathrm{~nm}$ results in a strong emission from curcumin in all gels (insets of Figure 12), indicating that curcumin is in hydrophobic domains of the systems network (near the gel fibres), as it does not emit fluorescence in aqueous environments. Highly similar quenching effect of the monomer band was obtained, and similar curcumin to monomer emission ratio was also verified. This demonstrates that the nanoparticles did not significantly affect the distance of curcumin relatively to the fibrils, as the quenching effect on the gels due to FRET from hydrogelator aromatic moieties to curcumin remains similar. However, it is observed that, for the three systems, curcumin emits fluorescence with different bandwidth, shape and wavelength maximum (Figure 12, insets). The magnetogel containing gold-decorated nanoparticles seems to have the stricter variety of environments for curcumin location, owing to its sharper emission band and blue-shifted fluorescence maximum. Thus, in this system, curcumin is restricted to more hydrophobic environments than in the magnetogel based on core/shell nanoparticles and in the hydrogel. Such hydrophobic environments might be associated with the long chain functional groups at the surface of the nanoparticles or changes on the fibres organization due to nanoparticle-fibre interactions.

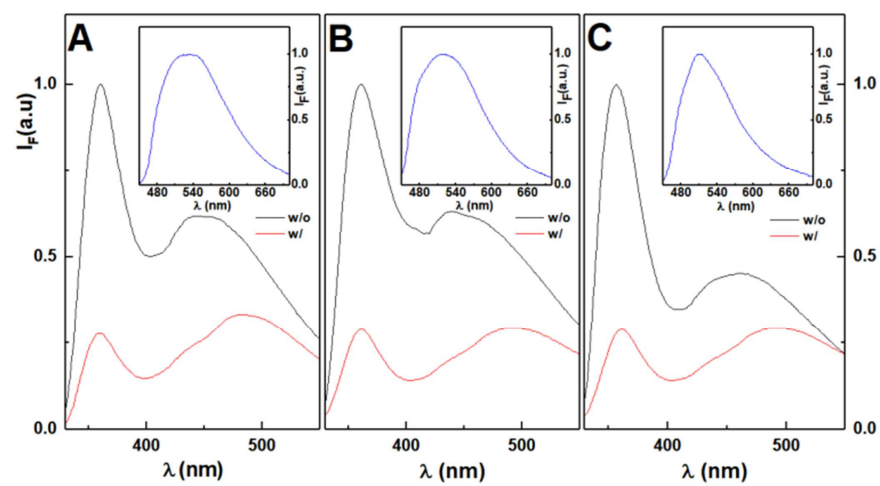

Figure 12. Fluorescence emission spectra of (A) Hydrogel, (B) Magnetogel with core/shell manganese ferrite/gold nanoparticles $(10 \mathrm{~m} / \mathrm{m} \%)$ and (C) Magnetogel with gold-decorated manganese ferrite nanoparticles (10 $\mathrm{m} / \mathrm{m} \%)$, with $(w /)$ and without $(w / 0)$ curcumin $\left(\lambda_{\text {exc }}=290 \mathrm{~nm}\right)$. Insets: Fluorescence emission spectra of directly excited curcumin $\left(\lambda_{\text {exc }}=420 \mathrm{~nm}\right)$.

Fluorescence anisotropy values of curcumin in the hydrogel $\left(r_{\mathrm{H}}=0.29\right)$ and magnetogels $\left(r_{\text {core/shell }}=0.11\right.$ and $\left.r_{\text {gold-decorated }}=0.25\right)$ demonstrate that the introduction of nanoparticles produced an increase on the fluidity of curcumin microenvironment, which can be associated with a different arrangement in the fibres promoted by the nanoparticles, especially in the system containing the core-shell ones. Moreover, the high anisotropy values evidence a high affinity of curcumin towards the matrix, even though it can also be related to more compact fibres and a denser network.

The hydrogelator fluorescence quantum yield was determined through eqn. (1), where a value of 0.041 was obtained. The calculated FRET efficiencies $\left(\Phi_{\text {FRET }}\right.$ ), Förster radius $\left(R_{0}\right)$, and donor-acceptor distances $\left(R_{D A}\right)$ are presented in Table 5 . The introduction of the nanoparticles did not produce a significant change on the distance between fibre aromatic groups and curcumin in all the nanosystems.

Table 5. FRET efficiency $\left(\Phi_{\text {FRET }}\right)$, Förster radius $\left(R_{0}\right)$ and donor-acceptor distances $\left(R_{D A}\right)$ from the interaction of curcumin with the hydrogel $(\mathrm{H})$, with magnetogel based on core/shell nanoparticles (CS) and with magnetogel containing gold-decorated nanoparticles (D).

\begin{tabular}{cccc}
\hline Nanosystem & $\boldsymbol{\Phi}_{\text {FRET }}$ & $\boldsymbol{R}_{\mathbf{0}}(\mathbf{n m})$ & $\boldsymbol{R}_{\text {DA }}(\mathbf{n m})$ \\
\hline H & 0.72 & 2.9 & 2.6 \\
CS & 0.73 & 2.9 & 2.4 \\
D & 0.73 & 2.8 & 2.4 \\
\hline
\end{tabular}

Drug delivery to biomembrane models. The delivery of curcumin into small unilamellar vesicles (SUVS) used as models of biomembranes was investigated by FRET, from curcumin (energy donor) to Nile Red (energy acceptor). Nile Red is a solvatochromic probe that intensely emits fluorescence in non-polar environments, but has almost negligible emission in water, the emission being accompanied by a blueshift with reduction of polarity. ${ }^{41-43,88}$ The high hydrophobic character of Nile Red leads to its preferential location in the lipid membranes, which can be explored to follow up the interaction between hydrogels/magnetogels and membrane models. Hereby, curcumin was loaded into the hydrogel or magnetogel and Nile Red into the SUVs. FRET can occur as evidenced by the overlap of curcumin fluorescence emission and Nile Red absorption (Figure S13 in Supplementary Information). Although Nile Red can be directly excited at $420 \mathrm{~nm}$ (curcumin excitation) such is highly inefficient, thus the increased fluorescence will be a result of the FRET process (Figure 13).

The interaction of magnetogel/hydrogel with membrane models can occur either by SUVs penetration into the hydrogel/magnetogel matrix, or through drug delivery. In the latter, the drug incorporated in the gel diffuses into the biomembranes. The gels might have small cavities, as no FRET was obtained in the hydrogel/magnetogel matrix, i.e. the biomembrane models did not penetrate the matrix. Nevertheless, the aqueous phase containing the added SUVs showed curcumin fluorescence emission and FRET to Nile Red about one hour after its addition. The curcumin emission after interaction is compared to the one of curcumin in SUVs, for the same final concentration (Figure 13).

The curcumin emission decrease (compared to SUVs containing only curcumin) and the enhanced Nile Red fluorescence indicate that drug delivery has occurred upon interaction between the gel and SUVs. Moreover, comparison with curcumin inside the hydrogel/magnetogel reveals that curcumin has localized to a more hydrophobic environment (biomembranes). Also, as the maximum emission wavelength of Nile Red after interaction is similar in SUVs, such suggests that the gel did not interfere on the organization of the biomembranes.

Donor-acceptor (curcumin-Nile Red) distances in SUVs were calculated from FRET data, using equations (3)-(6), being similar in all cases (Table 6). 


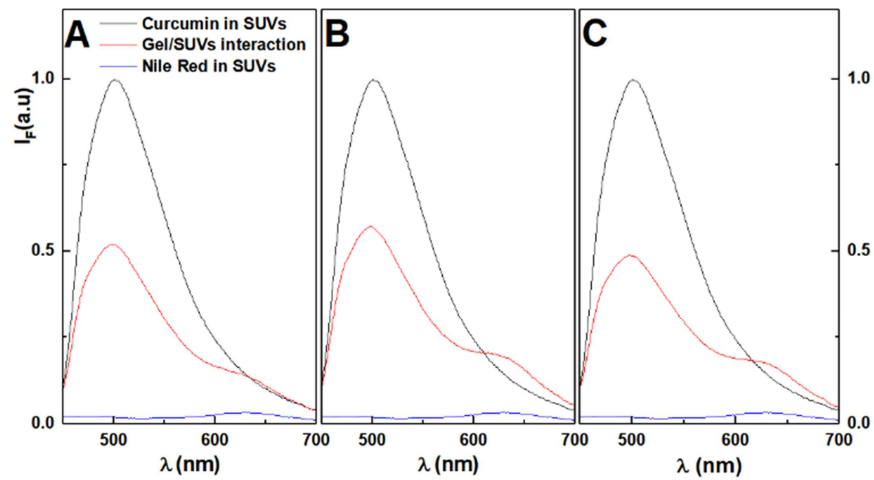

Figure 13. Fluorescence emission spectra $\left(\lambda_{\text {exc }}=420 \mathrm{~nm}\right)$ of SUVs of egg-PC/cholesterol 7:3 containing Nile Red, before and after interaction with curcumin-loaded hydrogel (A), magnetogel containing core/shell nanoparticles (B) and magnetogel containing gold-decorated manganese ferrite nanoparticles (C). Emission of curcumin in SUVs is also shown for comparison.

Table 6. FRET efficiency $\left(\Phi_{\text {FRET }}\right)$, Förster radius $\left(R_{0}\right)$ and donor-acceptor distances $\left(R_{D A}\right)$ between curcumin and Nile Red in SUVs, after drug delivery from the hydrogel $(H)$, the magnetogel based on core/shell nanoparticles (CS) and the magnetogel containing gold-decorated nanoparticles (D).

\begin{tabular}{cccc}
\hline Nanosystem & $\boldsymbol{\Phi}_{\text {FRET }}$ & $\boldsymbol{R}_{o}(\mathrm{~nm})$ & $\boldsymbol{R}_{\text {DA }}(\mathrm{nm})$ \\
\hline H & 0.46 & 2.6 & 2.7 \\
CS & 0.41 & 2.6 & 2.8 \\
D & 0.49 & 2.8 & 2.6 \\
\hline
\end{tabular}

Photothermia assays. For the study of photothermal ability, the emission of curcumin loaded in magnetogels was measured under irradiation (at $\lambda>600 \mathrm{~nm}$, to ensure the excitation of only the gold) along time. Upon irradiation, the absorbed light is converted into heat through non-radiative processes that are associated with the fast phase loss of the coherently excited electrons. ${ }^{31,32}$ The heat generated by the nanoparticles will be transferred to the medium, which is accompanied by the formation of vapor nanobubbles, where the expansion occurs until cooling and the collapse leads to a stress wave that propagates in the surrounding medium. ${ }^{89}$ Hereby, curcumin was used as a fluorescence thermometer to determine the mean temperature in the vicinity of the nanoparticles surface when embedded inside the hydrogel matrix. If the fluorescence quantum yield of curcumin close to the nanoparticles is negligible, then the dependence of the fluorescence intensity $\left(I_{\mathrm{F}}\right)$ on temperature can be expressed as eqn. (7) (see detailed explanation in Supplementary Information):

$$
\frac{I_{\mathrm{F}}^{0}}{I_{\mathrm{F}}} \propto A e^{-\left(E_{a} / R T\right)}
$$

where $I_{\mathrm{F}}^{0}$ is the fluorescence intensity without irradiation, $A$ is a pre-exponential constant and $E_{a}$ is the activation energy. ${ }^{90}$ Accordingly, for the curcumin-loaded hydrogel, the plot of logarithm of $\left(I_{\mathrm{F}}^{0} / I_{\mathrm{F}}\right)$ as a function of $1 / \mathrm{T}$ is linear, with a slope of $-61 \mathrm{~K}$ (Figure $\mathrm{S} 14$ in Supplementary Information), when temperature is changed from $20^{\circ} \mathrm{C}$ to $60^{\circ} \mathrm{C}$. The obtained logarithm of fluorescence intensity ratio for curcumin in the magnetogels subjected to continuous irradiation $(\lambda>600 \mathrm{~nm})$ allowed extrapolation of the nanoparticles surface temperature through the above determined parameter. The results qualitatively demonstrate that a heating effect is being produced upon continuous irradiation of the magnetogels (Figure 14). When the irradiation is stopped, a temperature decrease (fluorescence increase) is observed. Therefore, the heating effect is reversible, as demonstrated in a two cycle photothermia experiment in Figure 14B, even though the maximum temperature is slightly decreased in the second cycle. Hereby, it is expected that irradiation will enhance drug release from the magnetogel to the medium.

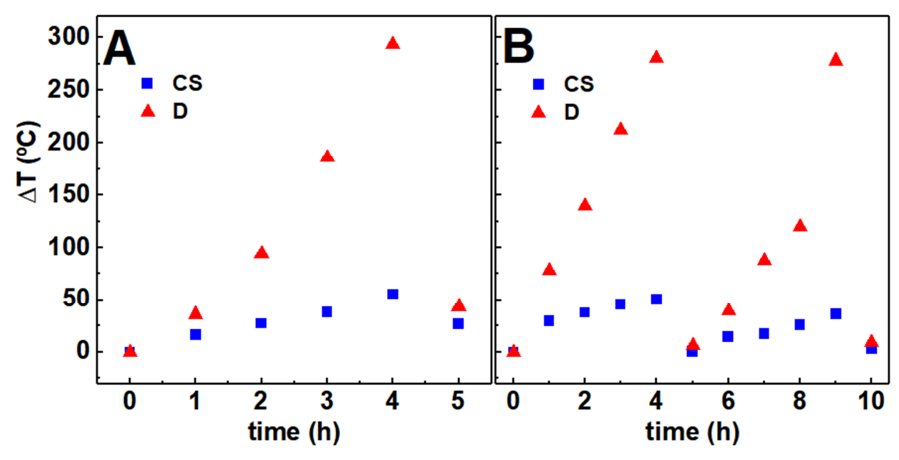

Figure 14. Temperature variation through irradiation of curcumin-loaded magnetogels containing core/shell manganese ferrite/gold nanoparticles (CS) and gold-decorated manganese ferrite nanoparticles (D) at $10 \mathrm{~m} / \mathrm{m} \%$ of nanoparticles. (A) First irradiation cycle. (B) Subsequent cycles of 5 hours.

Although quantitative information cannot be inferred owing to the several processes involved, the gold-decorated nanoparticles exhibit a high potential for photothermia. Such might be associated with the high gold nanoparticle concentration and the surface functionalization that favours a larger accumulation of curcumin around the nanoparticle cluster and higher temperatures, as heat will have a higher difficulty to dissipate than in the non-functionalized core/shell nanoparticles.

However, thermal degradation of curcumin must not be excluded (which further reduces fluorescence), besides the increased nonradiative relaxation rate by the heating of gold nanoparticles. The thermal degradation of curcumin results in the formation of vanillin, ferulic acid and 4-vynil guaiacol that are reported to have biological activity (antioxidant and anti-inflammatory). ${ }^{91}$

Drug release assays. Curcumin release from hydrogel and magnetogels was evaluated with and without excitation of the nanoparticles plasmon band. The nanosystems exhibit an initial burst release, followed by a slower release phase, where a similar behaviour was observed either in hydrogel and irradiated magnetogels on the first eight hours. The obtained results for plasmonic magnetogels were also compared to magnetogels containing non-coated manganese ferrite nanoparticles, to assess the influence of the gold coating on drug release. An interaction between curcumin and gold-containing nanoparticles is evidenced by the drug release profiles (Figure 15), as a lower curcumin concentration and slower drug release kinetics are observed for the non-irradiated magnetogels, while a higher release was obtained for the manganese ferrite containing magnetogels and hydrogel. 


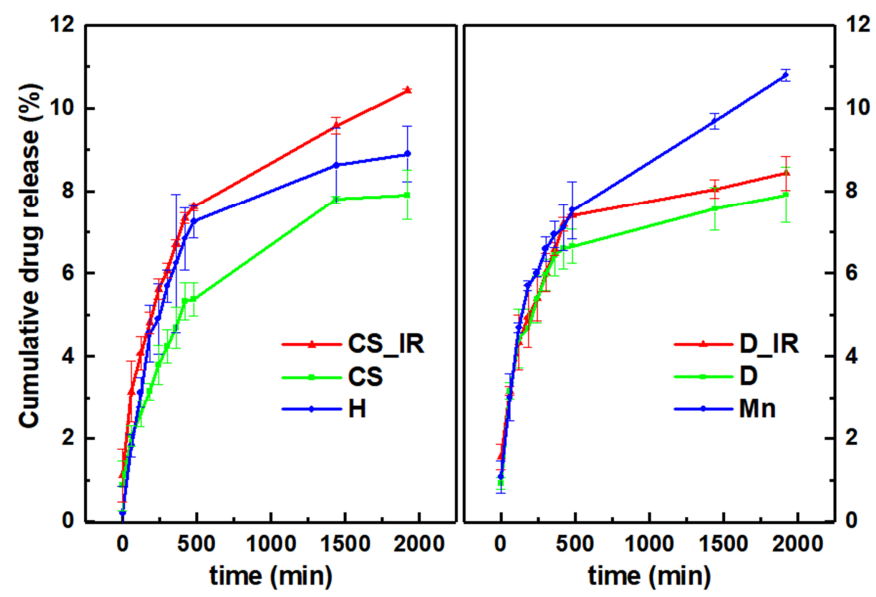

Figure 15. Cumulative drug release profiles (3 replicates) of curcumin $(20 \mu \mathrm{M})$ in hydrogel $(\mathrm{H})$, magnetogel containing $10 \%$ core/shell $\mathrm{MnFe}_{2} \mathrm{O}_{4} / \mathrm{Au}$ nanoparticles (CS), magnetogel with $10 \%$ gold-decorated $\mathrm{MnFe}_{2} \mathrm{O}_{4}$ nanoparticles (D), and magnetogel containing $10 \%$ non-coated $\mathrm{MnFe}_{2} \mathrm{O}_{4}$ nanoparticles $(\mathrm{Mn})$, with $(\mathrm{IR})$ and without incidence of radiation, for a contact area of $3.8 \mathrm{~cm}^{2}$.

Therefore, the results obtained with non-coated manganese ferrite nanoparticles suggest that the incorporation of nanoparticles opens the hydrogel pores and facilitates drug release, while nanoparticles capable of interacting with the loaded drug (core/shell and golddecorated nanoparticles) will difficult its release. The photothermia effect of core/shell nanoparticles on curcumin release is reflected by the enhanced release compared with the non-irradiated magnetogel. Hydrogel incubation in water at room temperature resulted in a $2 \%$ swelling increase, while incubation at $40{ }^{\circ} \mathrm{C}$ reduced the hydrogel swelling in $11 \%$. Thus, the drug release may be associated with the heating-induced gel contraction. However, the difference in drug release is small relatively to both the hydrogel and the magnetogel containing non-coated manganese ferrite nanoparticles. This profile is useful as the release of plasmonic magnetogels, even being lower when compared to the other systems (hydrogels and magnetogels containing magnetic nanoparticles), can be enhanced, making possible to control curcumin release. The interaction of the loaded drug with nanoparticles is more pronounced for the gold-decorated nanoparticles, where surface functionalization might have hindered drug release even with the use of photothermia.

To quantitatively evaluate the effect of nanoparticles and irradiation on the magnetogel, several mathematical models were fitted to the cumulative drug release profiles and the coefficients of determination were calculated (Table S1 in Supplementary Information). The first-order model is usually applied to the drug dissolution of water-soluble drugs in porous matrices. ${ }^{92,93}$ The Hixson-Crowell model describes the remaining concentration at time $t$ from a system that changes in surface area and diameter over time, but the initial geometrical form is kept constant. ${ }^{92}$ The Korsmeyer-Peppas model is applicable to polymeric systems that include both diffusion and erosion of polymer chains, which is described by a rate constant $K_{\mathrm{s}}$ that includes the structural and geometrical characteristics of the nanosystem and a coefficient $n$ that is related with the diffusion mechanism. ${ }^{92-95}$ The Higuchi model assumes that the drug concentration in the nanosystem is much higher than its solubility, drug diffusion occurs in a single dimension and diffusivity is constant, the drug particle size is much smaller than the nanosystem thickness, effects due to matrix swelling and dissolution are negligible, and that the release environment provides perfect sink conditions, i.e. the solvent volume is much larger than the saturated nanosystem solution. ${ }^{92,94}$ The Gompertz model adequately describes the release profiles where the drug has good solubility and intermediate release rates. ${ }^{92}$

Overall, the $R^{2}$ values (Table S1 in Supplementary Information) demonstrate that the results are not modelled by the first-order and the Hixson-Crowell model, i.e. the released drug (curcumin) is not water soluble and the hydrogel does not change in diameter overtime. The results are best fitted by a Gompertz model (eqn. 8),

$$
X_{t}=X_{\max } e^{-a e^{b \log _{10} t}}
$$

where $X_{t}$ and $X_{\text {max }}$ are the dissolved fraction percentages at time $t$ and its maximum, $a$ is a shape parameter and $b$ is the dissolution rate per unit of time. ${ }^{92}$

However, the Korsmeyer-Peppas model (eqn. 9) also suggests that the release can be associated to a diffusion-controlled mechanism,

$$
C_{t}=C_{0} K_{s} t^{n}
$$

where $C_{0}$ and $C_{t}$ are the concentrations at time 0 and $t$, and $K_{\mathrm{s}}$ is the rate constant. When $n<0.45$, the release mechanism is diffusion-controlled (Fickian release), $0.45<n<0.89$ indicates a combination of diffusion and erosion drug release (non-Fickian release), $0.89<n<1$ indicates a relaxation-controlled release, and if $n>1$, the release is controlled by swelling and polymer chain relaxation. $^{\text {92-95 }}$

The determined kinetic dissolution constants and parameters of these two models (eqn. 8 and 9) are presented in Table 7.

Table 7. Release coefficients of the Korsmeyer-Peppas and Gompertz models obtained for the curcumin release profiles $(20 \mu \mathrm{M})$ in hydrogel, magnetogel containing $10 \%$ core/shell manganese ferrite/gold nanoparticles (CS), magnetogels containing $10 \%$ gold-decorated nanoparticles (D), and magnetogels containing $10 \%$ non-coated manganese ferrite nanoparticles $\left(\mathrm{MnFe}_{2} \mathrm{O}_{4}\right)$, with (IR) and without incidence of radiation.

\begin{tabular}{ccc|ccc}
\hline & \multicolumn{3}{c}{ Korsmeyer-Peppas } & \multicolumn{3}{c}{ Gompertz } \\
\hline & $\boldsymbol{K}_{\mathbf{s}}$ & $\boldsymbol{n}$ & $\boldsymbol{X}_{\max }$ & $\boldsymbol{a}$ & $\boldsymbol{b}$ \\
\hline Hydrogel & 0.87 & 0.32 & 10.48 & 36.97 & 1.67 \\
CS & 0.51 & 0.37 & 18.89 & 8.11 & 0.69 \\
CS_IR & 1.99 & 0.21 & 18.89 & 7.25 & 0.76 \\
D & 3.93 & 0.21 & 9.25 & 11.23 & 1.30 \\
D_IR & 3.72 & 0.23 & 9.99 & 13.27 & 1.34 \\
MnFe $_{\mathbf{2}} \mathbf{O}_{4}$ & 1.18 & 0.29 & 18.96 & 6.54 & 0.73 \\
\hline
\end{tabular}

The Gompertz model parameters demonstrate that the nanoparticles in magnetogels hampered the release of curcumin comparatively to the hydrogel. This effect may be associated with either an interaction between curcumin and the nanoparticles, or the nanoparticles introducing inhomogeneities in the hydrogel matrix that difficult the drug diffusion through it. Moreover, the irradiated magnetogels demonstrated faster drug release kinetics, where the highest increase in $b$ coefficient is observed for the core/shell or the control with manganese ferrite nanoparticles. On 
the other hand, the lower increase in $b$ coefficient in the case of gold-decorated nanoparticles can be associated with the surface functionalization not favouring the release of curcumin.

The nanoparticles do show an impact on the predicted maximum curcumin dissolution that can be associated with a perturbation of free energy difference between the magnetogel matrix and the solution. The core/shell nanoparticles might induce a reduced affinity of curcumin towards the matrix, while the gold-decorated nanoparticles, due to the functionalized surface, might lead to a higher affinity of the hydrophobic drug towards the matrix.

Considering the potential anti-inflammatory activity of these nanosystems, release profiles of the hydrogelator compound were also assessed, with and without irradiation (Figure S15 and Table S2 in Supplementary Information). The nanoparticles did not severely affect the release of the hydrogelator. After gelation, a certain amount of free hydrogelator is in equilibrium with the aggregate state in the hydrogel matrix, which is released to the buffer. The release profiles were fitted with the previous described drug release models (Table S3 in Supplementary Information), showing that they cannot be described by the dissolution of a water-soluble drug or geometrical changes of the gel. The good fitting obtained with the other models suggests that the release mechanism can be described by a diffusion-controlled mechanism. The lack of agreement of Korsmeyer-Peppas model with the Gompertz model indicates that nanoparticles and irradiation do not significantly affect the hydrogelator release rate.

The irradiation influence on the release of curcumin towards biomembrane models (SUVS) was assessed by measuring the interaction during 8 hours. The hydrogel and magnetogels without irradiation showed a similar sigmoidal behaviour (Figure S16 in Supplementary Information), while the magnetogels subjected to irradiation exhibited an accelerated accumulation of curcumin in biomembrane models in the first two hours. Hereby, considering that saturation is due to curcumin attaining a partitioning equilibrium between the hydrogel/magnetogel and the limited number of available liposomes, the initial release acceleration (that was also observed in release to buffer) further demonstrates that photothermia might be used as a trigger to burst the release of the encapsulated drug in supramolecular magnetogels.

\section{Conclusions}

Considering the sulphur affinity towards gold, a hydrogelator comprising naproxen and a thioether, Npx-L-Met-Z- $\Delta$ Phe-OH, was synthesized and physicochemically characterized. The hydrogelator was computationally evaluated as a potential anti-inflammatory agent.

Magnetogels were successfully obtained for a limit of $10 \mathrm{~m} / \mathrm{m} \%$ of core/shell manganese ferrite/gold or gold-decorated manganese ferrite nanoparticles, above which sedimentation was faster than gelation. The nanoparticles induced an increase of random coil and a decrease of ordered structures, and no major effect over the chiral stacking of naproxen moieties.

The nanosystems showed to be suitable for encapsulation of the model drug curcumin. The presence of nanoparticles improved the encapsulation, as it induced less polar regions in the hydrogel matrix. Moreover, interaction of the gels with biomembrane models (SUVS) showed that curcumin can be delivered, but no penetration of vesicles into the gel matrix occur owing to the high density of fibres and small aqueous cavity size.

Gold-decorated manganese ferrite nanoparticles were more efficient in the generation of heat compared to the core/shell manganese ferrite/gold nanoparticles, according to the obtained results using curcumin as a fluorescence thermometer. However, drug release by irradiation was only enhanced in the core/shell nanoparticle-containing magnetogels, though delivery was higher in both irradiated gels compared to non-irradiated ones.

The superparamagnetic properties of the nanoparticles are suitable for biomedical applications and the gels elastic properties fall in the range of soft tissues. Therefore, the magnetogels based on Npx-L-Met-Z- $\triangle$ Phe-OH are promising for multimodal cancer therapy by combining controlled drug delivery and photothermia.

\section{Conflicts of interest}

There are no conflicts of interest to declare.

\section{Acknowledgements}

This work was supported by the Portuguese Foundation for Science and Technology (FCT) in the framework of the Strategic Funding of CF-UM-UP (UID/FIS/04650/2019) and CQUM (UID/QUI/00686/ 2019). FCT, FEDER, PORTUGAL2020 and COMPETE2020 are also acknowledged for funding under research projects PTDC/QUIQFI/28020/2017 (POCI-01-0145-FEDER-028020) and PTDC/QUIQOR/29015/2017 (POCl-01-0145-FEDER-029015).

The magnetic measurements were supported by projects UTAP-EXPL/NTec/0046/2017, NORTE-01-0145-FEDER-028538 and PTDC/FIS-MAC/29454/2017. Authors gratefully acknowledge Dr L. M. Vieira for carrying out the FTIR measurements at the Infrared Spectroscopy Lab. of the Centre of Physics, Univ. Minho.

\section{Notes and references}

1 P. Kumari, B. Ghosh and S. Biswas, Nanocarriers for cancertargeted drug delivery, J. Drug Target., 2015, 24, 179-191.

2 L. Torre, R. Siegel, E. Ward and A. Jemal, Global cancer incidence and mortality rates and trends - An update, Cancer Epidemiol. Biomark. Prev., 2015, 25, 16-27.

3 A. Jemal, F. Bray; M. Center, J. Ferlay, E. Ward and D. Forman, Global cancer statistics, CA: Cancer J. Clin., 2011, 61, 69-90.

4 Y. A. Lee and M. Hashibe, Tobacco, alcohol, and cancer in low and high income countries, Ann. Glob. Health, 2014, 80, 378-383.

5 N. Berger, Obesity and cancer pathogenesis, Ann. N. Y. Acad. Sci, 2014, 1311, 57-76.

6 H. Nagai and Y. Kim, Cancer prevention from the perspective of global cancer burden patterns, J. Thorac. Dis., 2017, 9, 448-451.

7 C. Huang, D. Ju, C. Chang, P. M. Reddy and B. Velmurugan, A review on the effects of current chemotherapy drugs and natural agents in treating non-small cell lung cancer, Biomedicine, 2017, 7, 12-23.

8 M. Ma, Y. Kuang, Y. Gao, Y. Zhang, P. Gao and B. Xu, Aromatic-aromatic interactions induce the self-assembly of pentapeptidic derivatives in water to form nanofibers and supramolecular hydrogels, J. Am. Chem. Soc., 2010, 132, 2719-2728. 
9 S. Sutton, N. L. Campbell, A. I. Cooper, M. Kirkland, W. J. Frith and D. J. Adams, Controlled release from modified amino acid hydrogels governed by molecular size or network dynamics, Langmuir, 2009, 25, 10285-10291.

10 E. Caló and V. V. Khutoryanskiy, Biomedical applications of hydrogels: A review of patents and commercial products, Eur. Polym. J., 2015, 65, 252-267.

11 H. Vilaça, T. Castro, F. M. G. Costa, M. Melle-Franco, L. Hilliou, I. W. Hamley, E. M. S. Castanheira, J. A. Martins and P. M. T. Ferreira, Self-assembled RGD dehydropeptide hydrogels for drug delivery applications, J. Mater. Chem. B, 2017, 5, 8607-8617.

12 A. Bonhome-Espinosa, F. Campos, I. Rodriguez, V. Carriel, J. Marins, A. Zubarev, J. Duran and M. Lopez-Lopez, Effect of particle concentration on the microstructural and macromechanical properties of biocompatible magnetic hydrogels, Soft Matter, 2017, 13, 2928-2941

$13 \mathrm{Y}$. Li, F. Wang and H. Cui, Peptide-based supramolecular hydrogels for delivery of biologics, Bioeng. Transl. Med., 2016, 1, 306-322.

14 S. Bhattacharya and S. Samanta, Soft-nanocomposites of nanoparticles and nanocarbons with supramolecular and polymer gels and their applications, Chem. Rev., 2016, 116, 11967-12028.

$15 \mathrm{M}$. Antman-Passig and O. Shefi, Remote magnetic orientation of 3D collagen hydrogels for directed neuronal regeneration, Nano Lett., 2016, 16, 2567-2573.

16 S. Salatin, J. Barar, M. Barzegar-Jalali, K. Adibkia, M. Milani and $M$. Jelvehgari, Hydrogel nanoparticles and nanocomposites for nasal drug/vaccine delivery, Arch. Pharm. Res., 2016, 39, 1181-1192.

17 A. Z. Cardoso, A. E. A. Alvarez, B. N. Cattoz, P. C. Griffiths, S. M. King, W. J. Frith and D. J. Adams, The influence of the kinetics of self-assembly on the properties of dipeptide hydrogels, Faraday Discuss., 2013, 166, 101-116.

18 G. Fichman and E. Gazit, Self-assembly of short peptides to form hydrogels: Design of building blocks, physical properties and technological applications, Acta Biomater., 2014, 10, 1671-1682.

19 O. Ozay, S. Ekici, Y. Baran, N. Aktas and N. Sahiner, Removal of toxic metal ions with magnetic hydrogels., Water Res., 2009, 43, 4403-4411.

20 H. Kim, A. Jo, S. Baek, D. Lim, S. Park, S. Cho, J. Chung and J. Yoon, Synergistically enhanced selective intracellular uptake of anticancer drug carrier comprising folic acid-conjugated hydrogels containing magnetite nanoparticles, Sci. Rep., 2017, 7, 1-10.

21 A. Hervault and N. Thanh, Magnetic nanoparticle-based therapeutic agents for thermo-chemotherapy treatment of cancer, Nanoscale, 2014, 6, 11553-11573.

22 A. Akbarzadeh, M. Samiei and S. Davaran, Magnetic nanoparticles: Preparation, physical properties, and applications in biomedicine, Nanoscale Res. Lett., 2012, 7, 144.

23 S. R. S. Veloso, P. M.T. Ferreira, J.A. Martins, P. J. G. Coutinho and E. M. S. Castanheira, Magnetogels: Prospects and main challenges in biomedical applications, Pharmaceutics, 2018, 10, 145.

24 Z. Q. Zhang and S. C. Song, Thermosensitive/superparamagnetic iron oxide nanoparticle-loaded nanocapsule hydrogels for multiple cancer hyperthermia, Biomaterials, 2016, 106, 13-23.

25 B. Issa, I. M. Obaidat, B. A. Albiss and Y. Haik, Magnetic nanoparticles: Surface effects and properties related to biomedicine applications, Int. J. Mol. Sci., 2013, 14, 2126621305.

26 L. I. Cabrera, A. Somoza, J. F. Marco, C. J. Serna and M. P. Morales, Synthesis and surface modification of uniform
$\mathrm{MFe}_{2} \mathrm{O}_{4}(\mathrm{M}=\mathrm{Fe}, \mathrm{Mn}$, and $\mathrm{Co}$ ) nanoparticles with tunable sizes and functionalities, J. Nanopart. Res., 2012, 14, 873.

27 M. Colombo, S. Carregal-Romero, M. F. Casula, L. Gutiérrez, M. P. Morales, I. B. Böhm, J. T. Heverhagen, D. Prosperi and W.J. Parak, Biological applications of magnetic nanoparticles, Chem. Soc. Rev., 2012, 41, 4306-4334.

28 M. Das, K. Shim, S. An and D. Yi, Review on gold nanoparticles and their applications, Toxicol. Environ. Health Sci., 2011, 3, 193-205.

29 M. H. Carneiro and F. Barbosa, Gold nanoparticles: A critical review of therapeutic applications and toxicological aspects, J. Toxicol. Environ. Health, Part B, 2016, 19, 129-148.

30 Y. Yeh, B. Creran and V. Rotello, Gold nanoparticles: Preparation, properties, and applications in bionanotechnology, Nanoscale, 2012, 4, 1871-1880.

31 X. Huang and M. El-Sayed, Gold nanoparticles: Optical properties and implementations in cancer diagnosis and photothermal therapy, J. Adv. Res., 2010, 1, 13-28.

$32 \mathrm{E}$. Elsherbini, Laser and radiofrequency-induced hyperthermia treatment via gold-coated magnetic nanocomposites, Int. J. Nanomed., 2011, 6, 2155-2165.

33 J. Li, Y. Kuang, Y. Gao, X. Du, J. Shi and B. Xu, d-Amino acids boost the selectivity and confer supramolecular hydrogels of a nonsteroidal anti-inflammatory drug (NSAID), J. Am. Chem. Soc., 2013, 135, 542-545.

34 H. Vilaça, G. Pereira, T. G. Castro, B. F. Hermenegildo, J. Shi, T. Q. Faria, N. Micaêlo, R. M. M. Brito, B. Xu, E. M. S. Castanheira, J. A. Martins and P. M. T. Ferreira, New selfassembled supramolecular hydrogels based on dehydropeptides, J. Mater. Chem. B, 2015, 3, 6355-6367.

35 O. Keller, W. E. Keller, G. van Look and G. Wersin, Tertbutoxycarbonylation of amino acids and their derivatives: $n$ tert-butoxycarbonyl-L-phenylalanine, Org. Synth., 1985, 63, 160.

36 H. Vilaça, A.C.L. Hortelão, E.M.S. Castanheira, M.J.R.P. Queiroz, L. Hilliou, I. Hamley, J. A. Martins and P. M. T. Ferreira, Dehydrodipeptide hydrogelators containing naproxen $N$-capped tryptophan: self-assembly, hydrogel characterization, and evaluation as potential drug nanocarriers, Biomacromolecules, 2015, 16, 3562-3573.

37 A. R. O. Rodrigues, J. M. F. Ramos, I. T. Gomes, B. G. Almeida, J. P. Araújo, M. J. R. P. Queiroz, P. J. G. Coutinho and E. M. S. Castanheira, Magnetoliposomes based on manganese ferrite nanoparticles as nanocarriers for antitumor drugs, RSC Adv., 2016, 6, 17302-17313.

38 A. R. O. Rodrigues, J. O. G. Matos, A. M. Nova Dias, B. G. Almeida, A. Pires, A.M. Pereira, J.P. Araújo, M.J.R.P. Queiroz, E. M. S. Castanheira and P. J. G. Coutinho, Development of Multifunctional Liposomes Containing Magnetic/Plasmonic $\mathrm{MnFe}_{2} \mathrm{O}_{4} / \mathrm{Au}$ Core/Shell Nanoparticles, Pharmaceutics, 2019, 11, 10.

39 S. Liu, T. Zhu, Y. Wang and Z. Liu, Synthesis and pH Dependent Optical Properties of Gold Nanoparticles Capped with Mercaptopropionic Acid, Mol. Cryst. Liq. Cryst. Sci. Technol. A, 1999, 337, 245-248.

40 D. Perrin and B. Dempsey, Buffers for $\mathrm{pH}$ and Metal Ion Control, London, England, Chapman and Hall, 1974.

41 P. Greenspan and S. Fowler, Spectrofluorometric studies of the lipid probe Nile Red, J. Lipid Res., 1985, 26, 781-789.

$42 \mathrm{I}$. and G. Krishnamoorthy, Probing the link between proton transport and water content in lipid membranes. J. Phys. Chem. B, 2001, 105, 1484-1488.

43 P. J. G. Coutinho, E.M.S. Castanheira, M.C. Rei and M.E.C.D.R. Oliveira, Nile Red and DCM fluorescence anisotropy studies in $\mathrm{C}_{12} \mathrm{E}_{7}$ /DPPC Mixed Systems, J. Phys. Chem. B, 2002, 106, 12841-12846. 
44 G. A. Crosby and J. N. Demas, The measurement of photoluminescence quantum yields. - Review, J. Phys. Chem., 1971, 75, 991-1024.

$45 \mathrm{~S}$. Fery-Forgues and D. Lavabre, Are fluorescence quantum yields so tricky to measure? A demonstration using familiar stationery products, J. Chem. Educ., 1999, 76, 1260-1264.

46 E. P. Kirby and R. F. Steiner, Influence of solvent and temperature upon the fluorescence of indole derivatives, $J$. Phys. Chem., 1970, 74, 4480-4490.

47 B. Valeur, Molecular fluorescence - Principles and Applications, Wiley-VCH, Weinheim, 2001.

$48 \mathrm{~S}$. Lin and $\mathrm{H}$. Chu, Fourier transform infrared spectroscopy used to evidence the prevention of $\beta$-sheet formation of amyloid $\beta(1-40)$ peptide by a short amyloid fragment, Int. J. Biol. Macromol., 2003, 32, 173-177.

49 H. Fabian and W. Mäntele, Infrared spectroscopy of proteins. In Handbook of Vibrational Spectroscopy (eds. J.M. Chalmers and P. R. Griffiths), 2006. Retrieved Nov. 6 2018, from https://doi.org/10.1002/0470027320.s8201

$50 \mathrm{H}$. Fabian and C. P. Schultz, Fourier transform infrared spectroscopy in peptide and protein analysis. In Encyclopedia of Analytical Chemistry (eds. R. A. Meyers and C. Schöneich), 2006. Retrieved Nov. 6 2018, from https://doi.org/10.1002/9780470027318.a1612

51 B. H. Stuart, Infrared spectroscopy of biological applications: An overview. In Encyclopedia of Analytical Chemistry (eds. R. A. Meyers and C. Schöneich), 2012. Retrieved Nov. 6 2018, from https://doi.org/10.1002/9780470027318.a0208.pub2

$52 \mathrm{H}$. Yang, S. Yang, J. Kong, A. Dong and S. Yu, Obtaining information about protein secondary structures in aqueous solution using Fourier transform IR spectroscopy, Nat. Protoc., 2015, 10, 382-396.

53 Gaussian 09, Revision A.02, M. J. Frisch, G. W. Trucks, H. B. Schlegel, G. E. Scuseria, M. A. Robb, J. R. Cheeseman, G. Scalmani, V. Barone, B. Mennucci, G. A. Petersson, H. Nakatsuji, M. Caricato, X. Li, H. P. Hratchian, A. F. Izmaylov, J. Bloino, G. Zheng, J. L. Sonnenberg, M. Hada, M. Ehara, K. Toyota, R. Fukuda, J. Hasegawa, M. Ishida, T. Nakajima, Y. Honda, O. Kitao, H. Nakai, T. Vreven, J. A. Montgomery Jr., J. E. Peralta, F. Ogliaro, M. Bearpark, J. J. Heyd, E. Brothers, K. N. Kudin, V. N. Staroverov, R. Kobayashi, J. Normand, K. Raghavachari, A. Rendell, J. C. Burant, S. S. Iyengar, J. Tomasi, M. Cossi, N. Rega, J. M. Millam, M. Klene, J. E. Knox, J. B. Cross, V. Bakken, C. Adamo, J. Jaramillo, R. Gomperts, R. E. Stratmann, O. Yazyev, A. J. Austin, R. Cammi, C. Pomelli, J. W. Ochterski, R. L. Martin, K. Morokuma, V. G. Zakrzewski, G. A. Voth, P. Salvador, J.J. Dannenberg, S. Dapprich, A. D. Daniels, Ö. Farkas, J. B. Foresman, J. V. Ortiz, J. Cioslowski, D. J. Fox, Gaussian, Inc., Wallingford CT, 2009.

54 D. M. Pereira, G. Correia-da-Silva, P. Valentão, N. Teixeira and P. B. Andrade, Palmitic acid and Ergosta-7,22-dien-3-ol contribute to the apoptotic effect and cell cycle arrest of an extract from Marthasterias glacialis L. in Neuroblastoma cells, Mar. Drugs, 2014, 12, 54-68.

$55 \mathrm{E}$. Valeur and M. Bradley, Amide bond formation: beyond the myth of coupling reagents, Chem. Soc. Rev., 2009, 38, 606631.

56 P. M. T. Ferreira, L. S. Monteiro, G. Pereira, L. Ribeiro, J. Sacramento and L. Silva, Reactivity of dehydroamino acids and dehydrodipeptides towards $\mathrm{N}$-bromosuccinimide: Synthesis of $\beta$-bromo- and $\beta, \beta$-dibromodehydroamino acid derivatives and of substituted 4 -imidazolidinones, Eur. J. Org. Chem. 2007, 2007, 5934-5949.

57 M. Velazquez, M. Valero, L. J. Rodríguez, S. M. B. Costa and M. A. Santos, Hydrogen bonding in a non-steroidal antiinflammatory drug Naproxen, J. Photochem. Photobiol. B: Biol., 1995, 29, 23-31.
58 E. Yeargers and L. Augenstein, UV spectral properties of phenylalanine powder, Biophys. J., 1965, 5, 687-696.

59 S. R. S. Veloso, C. A. B. Magalhães, A. R. O. Rodrigues, H. Vilaça, M. J. R. P. Queiroz, J. A. Martins, P. J. G. Coutinho, P. M.T. Ferreira and E.M.S. Castanheira, Novel dehydropeptidebased magnetogels containing manganese ferrite nanoparticles as antitumor drug nanocarriers, Phys. Chem. Chem. Phys., 2019, 21, 10377-10390.

60 H. Azizian, Z. Mousavi, H. Faraji, M. Tajik, K. Bagherzadeh, P. Bayat, A. Shafiee and A. Almasirad, Arylhydrazone derivatives of naproxen as new analgesic and antiinflammatory agents: Design, synthesis and molecular docking studies, J. Mol. Graph. Model., 2016, 67, 127-136.

61 E. Kwizera, E. Chaffin, Y. Wang and X. Huang, Synthesis and properties of magnetic-optical core-shell nanoparticles, RSC Adv., 2017, 7, 17137-17153.

62 M. Y. Rafique, P. Li-Qing, Q. Javed, M. Z. Iqbal, Q. Hong-Mei, M. H. Farooq, G. Zhen-Gang and M. Tanveer, Growth of monodisperse nanospheres of $\mathrm{MnFe}_{2} \mathrm{O}_{4}$ with enhanced magnetic and optical properties, Chinese Phys. B, 2013, 22, 107101.

63 E. Chaffin, S. Bhana, R. O'Connor, X. Huang and Y. Wang, Impact of core dielectric properties on the localized surface plasmonic spectra of gold-coated magnetic core-shell nanoparticles, J. Phys. Chem. B, 2014, 118, 14076-14084.

64 S. Link and M. A. El-Sayed, Size and temperature dependence of the plasmon absorption of colloidal gold nanoparticles, $J$. Phys. Chem. B, 1999, 103, 4212-4217.

65 K.-S. Lee and M. A. El-Sayed, Gold and silver nanoparticles in sensing and imaging: Sensitivity of plasmon response to size, shape, and metal composition, J. Phys. Chem. B, 2006, 110, 19220-19225.

66 Y. Bagbi, A. Sarswat, D. Mohan, A. Pandey and P. R. Solanki, Lead and Chromium adsorption from water using L-Cysteine functionalized magnetite $\left(\mathrm{Fe}_{3} \mathrm{O}_{4}\right)$ nanoparticles, Sci. Reports, 2017, 7, 7672.

$67 \mathrm{~T}$. Sen and A. Patra, Resonance energy transfer from rhodamine $6 \mathrm{G}$ to gold nanoparticles by steady-state and time-resolved spectroscopy, J. Phys. Chem. C, 2008, 112, 3216-3222.

$68 \mathrm{~J}$. Rodriguez-Carvajal, Recent advances in magnetic structure determination by neutron powder diffraction, Physica $B$, 1993, 192, 55-69.

69 Z. Xu, Y. Hou and S. Sun, Magnetic core/shell $\mathrm{Fe}_{3} \mathrm{O}_{4} / \mathrm{Au}$ and $\mathrm{Fe}_{3} \mathrm{O}_{4} / \mathrm{Au} / \mathrm{Ag}$ nanoparticles with tunable plasmonic properties, J. Am. Chem. Soc., 2007, 129, 8698-8699.

70 L. Wang, J. Luo, M. M. Maye, Q. Fan, Q. Rendeng, M. H. Engelhard, C. Wang, Y. Lin and C.-J. Zhong, Iron oxide-gold core-shell nanoparticles and thin film assembly, J. Mater. Chem., 2005, 15, 1821-1832.

71 M. Mandal, S. Kundu, S. K. Ghosh, S. Panigrahi, T. K. Sau, S. M. Yusuf and T. Pal, Magnetite nanoparticles with tunable gold or silver shell, J. Coll. Interface Sci., 2005, 286, 187-194.

72 J. Liu, H. Zheng, P. Poh, H. Machens and A. Schilling, Hydrogels for engineering of perfusable vascular networks, Int. J. Mol. Sci., 2015, 16, 15997-16016.

73 Z. Yang, Y. Hemar, L. Hilliou, E. Gilbert, D. McGillivray, M. Williams and $\mathrm{S}$. Chaieb, Nonlinear behavior of gelatin networks reveals a hierarchical structure, Biomacromolecules, 2015, 17, 590-600.

74 A. Dobrynin and J. Carrillo, Universality in nonlinear elasticity of biological and polymeric networks and gels, Macromolecules, 2011, 44, 140-146.

75 F. Meng and E. Terentjev, Nonlinear elasticity of semiflexible filament networks, Soft Matter, 2016, 12, 6749-6756.

76 T. Gisler, R. C. Ball and D. A. Weitz, Strain hardening of fractal colloidal gels, Phys. Rev. Lett., 1999, 82, 1064-1067. 
77 X. Fan, H. Xu, Q. Zhang, D. Xiao, Y. Song and Q. Zheng, Insight into the weak strain overshoot of carbon black filled natural rubber, Polymer, 2019, 167, 109-117.

78 M. Chekini, R. Filter, J. Bierwagen, A. Cunningham, C. Rockstuhl and T. Bürgi, Fluorescence enhancement in largescale self-assembled gold nanoparticle double arrays, J. Appl. Phys., 2015, 118, 1-10.

79 T. Nakamura and S. Hayashi, Enhancement of dye fluorescence by gold nanoparticles: Analysis of particle size dependence, Jpn. J. App. Phys., 2005, 44, 6833-6837.

80 J. Chen, Y. Jin, N. Fahruddin and J. Zhao, Development of gold nanoparticle-enhanced fluorescent nanocomposites, Langmuir, 2013, 29, 1584-1591.

81 P. A. Presta and M. J. Stillman, Circular dichroism (CD) spectroscopy. In Encyclopedia of Inorganic and Bioinorganic Chemistry (ed. R. A. Scott), 2011. Retrieved Jan. 5 2019, from https://doi.org/10.1002/9781119951438.eibc0308

82 A. Urbach, Circular dichroism spectroscopy in the undergraduate curriculum, J. Chem. Educ., 2010, 87, 891893.

83 N. Greenfield, Applications of circular dichroism in protein and peptide analysis, Trends Anal. Chem., 1999, 18, 236-244.

84 S. M. Kelly, T. J. Jess and N. C. Price, How to study proteins by circular dichroism, Biochim. Biophys. Acta, 2005, 1751, 119 139.

85 K. L. Morris, L. Chen, A. Rodger, D. J. Adams and L. C. Serpell, Structural determinants in a library of low molecular weight gelators, Soft Matter, 2015, 11, 1174-1181.

86 J. Smit, Magnetic Properties of Materials, McGraw Hill, New York, USA, 1971.

87 C. Chignell, P. Bilskj, K. Reszka, A. Motten, R. Sik and T. Dahl, Spectral and photochemical properties of curcumin, Photochem. Photobiol., 1994, 59, 295-302.
88 G. Hungerford, E. M. S. Castanheira, M. E. C. D. Real Oliveira, M. G. Miguel and H. Burrows, Monitoring ternary systems of $\mathrm{C}_{12} \mathrm{E}_{5} /$ water/tetradecane via the fluorescence of solvatochromic probes, J. Phys. Chem. B, 2002, 106, 4061-4069.

89 V. Amendola, R. Pilot, M. Frasconi, O. Maragò and M. latì, Surface plasmon resonance in gold nanoparticles: a review, J. Phys. Cond. Matter, 2017, 29, 1-48.

90 K. Dahl, R. Biswas and M. Maroncelli, The photophysics and dynamics of diphenylbutadiene in alkane and perfluoroalkane solvents, J. Phys. Chem. B, 2003, 107, 78387853.

91 T. Esatbeyoglu, K. Ulbrich, C. Rehberg, S. Rohn and G. Rimbach, Thermal stability, antioxidant, and antiinflammatory activity of curcumin and its degradation product 4-vinyl guaiacol, Food Funct., 2015, 6, 887-893.

92 S. Dash, P. N. Murthy, L. Nath and P. Chowdhury, Kinetic modelling on drug release from controlled drug delivery systems, Acta Pol. Pharm., 2010, 67, 217-223.

93 J. Ghitman, R. Stan, A. Ghebaur, S. Cecoltan, E. Vasile and H. lovu, Novel PEG-modified hybrid PLGA-vegetable oils nanostructured carriers for improving performances of indomethacin delivery, Polymers, 2018, 10, 579.

94 S. Kini, D. Bahadur and D. Panda, Mechanism of anti-cancer activity of benomyl loaded nanoparticles in multidrug resistant cancer cells, J. Biomed. Nanotechnol., 2015, 11, 877-889.

95 M. Joy, S. Iyengar, J. Chakraborty and S. Ghosh, Layered double hydroxide using hydrothermal treatment: morphology evolution, intercalation and release kinetics of diclofenac sodium, Front. Mater. Sci., 2017, 11, 395-408. 